\title{
RESIDUAL GENERATOR FUZZY IDENTIFICATION FOR AUTOMOTIVE DIESEL ENGINE FAULT DIAGNOSIS
}

\author{
SILVIO SIMANI \\ Department of Engineering \\ University of Ferrara, Via Saragat 1E, 44124 Ferrara (FE), Italy \\ e-mail: silvio.simani@unife.it
}

\begin{abstract}
Safety in dynamic processes is a concern of rising importance, especially if people would be endangered by serious system failure. Moreover, as the control devices which are now exploited to improve the overall performance of processes include both sophisticated control strategies and complex hardware (input-output sensors, actuators, components and processing units), there is an increased probability of faults. As a direct consequence of this, automatic supervision systems should be taken into account to diagnose malfunctions as early as possible. One of the most promising methods for solving this problem relies on the analytical redundancy approach, in which residual signals are generated. If a fault occurs, these residual signals are used to diagnose the malfunction. This paper is focused on fuzzy identification oriented to the design of a bank of fuzzy estimators for fault detection and isolation. The problem is treated in its different aspects covering the model structure, the parameter identification method, the residual generation technique, and the fault diagnosis strategy. The case study of a real diesel engine is considered in order to demonstrate the effectiveness the proposed methodology.
\end{abstract}

Keywords: fault detection and isolation, analytical redundancy, Takagi-Sugeno fuzzy prototypes, residual generator fuzzy modelling and identification, real diesel engine.

\section{Introduction}

The control devices currently in use to improve the overall performance of industrial processes involve both sophisticated digital control techniques and complex hardware (sensors, actuators and processing units). The complexity means that the probability of fault occurrence can be significant and an automatic supervisory control system should be used to detect and isolate anomalous working conditions as early as possible. These motivations pushed great attention on Fault Detection and Isolation (FDI) in dynamic processes and a wide variety of approaches have been proposed (Chen and Patton, 1999; Isermann, 2005; Simani et al., 2003; Ding, 2008). They are based, e.g., on the parity space, state estimation, Unknown Input Observers (UIOs), Kalman Filters (KFs), Unknown Input Kalman Filters (UIKFs), and parameter identification. On the other hand, artificial intelligence techniques can be also exploited (Korbicz et al., 2004). Although many linear and nonlinear approaches have been developed, robust and reliable FDI for dynamic processes is still a problem open to further research.

In order to guarantee that faults can be detected and isolated (and distinguishable), accurate mathematical models of the process under investigation are required, in either the state space or input-output forms. Residuals should then be processed to detect an actual fault condition, rejecting any false alarms caused by noise or spurious signals. However, in practical situations, the straightforward application of model-based FDI techniques can be difficult, due to dynamic model complexity. In fact, the plant analytical description is usually designed to carefully capture all kinds of details relevant to the analysis and deployment of the real system. On the other hand, this intrinsic complexity makes the use of many cited linear FDI methods, almost infeasible and a viable procedure for practical application of FDI techniques is really necessary in practical cases (Chen and Patton, 1999; Simani et al., 2003).

In particular, many papers on model-based FDI were published over the last decade, using both signaland process model-based methods (Svard-Nyberg, 2010a; 2010b; Jain et al., 2012; Marusak and Tatjewski, 2008). Unsurprisingly, these show that the more accurate the model is at describing the engine behaviour, the better its performance will be in detecting anomalous conditions. Unfortunately, an accurate and complete mathematical 
model of such a complex thermodynamic system is usually unavailable, typically because of the assumptions introduced to reduce mathematical complexity. Hence, FDI schemes that relate to first principle engine models are costly to develop, while current alternatives tend to be mathematically complex or require considerable a priori knowledge to be incorporated into the monitoring scheme.

In this paper, the use of fuzzy identification is proposed through a real process for finding a viable solution to the FDI problem. To this end, two practical aspects of the presented work are stressed. Firstly, the system complexity may not indicate a requirement for a sophisticated physical or thermodynamic model. In fact, as shown in this work, a fuzzy identification method can be successfully used, thus obviating the requirement for physical models. In particular, the Errors-In-Variables (EIV) framework (Van Huffel and Lemmerling, 2002) and a proper identification algorithm are used in connection with fuzzy logic descriptions. Secondly, fuzzy prototypes for residual generation are considered instead of using purely nonlinear observers or filters. Moreover, as the purpose of system supervision is to monitor the conditions of the system at different working points, piecewise affine prototypes are successfully proposed. Real data from a diesel engine are considered.

This paper suggests to use fuzzy system theory, since it seems to be a natural tool to handle complicated and uncertain processes, such as diesel engines. Thus, it is suggested to exploit residual generators in the form of Takagi-Sugeno (TS) fuzzy prototypes (Takagi and Sugeno, 1985), whose parameters are easily obtained by identification procedures. It should finally be pointed out how the fuzzy approach can solve the problem at two levels. First, fuzzy TS models are used to generate residual signals for fault detection. Second, fault isolation is achieved using again the identified fuzzy TS prototypes organised into a bank structure.

Finally, the effectiveness of the proposed identification and fault diagnosis strategies is assessed on real data sequences acquired from full European driving cycle tests. Realistic fault conditions and different working situations have been considered, in order to provide an accurate validation of the proposed methodology. The main features of the diagnosis approach suggested in this paper are compared with different schemes based, e.g., on a UIO/KF bank.

The paper has the following structure. Section 2 briefly recalls the structure of the diesel engine. Section 4 addresses the fuzzy identification strategy exploited for obtaining the residual generators which will be used for the design of the fault diagnosis strategy. This diagnosis methodology is presented in Section 5. The achieved results summarised in Section 6 show the performances of the diagnosis schemes, validated on real data directly acquired from the diesel engine, and compared also with a different fault diagnosis strategy. Finally, Section 7 concludes the paper by highlighting the main achievements of the work.

\section{Diesel engine system}

Section 3 provides brief details regarding the diesel engine considered in this work, which was designed by the company VM Motors S.p.A. in connection with the embedded controller developed by BOSCH (Bosch, 2006). Section 3.1 illustrates the faults of interest for VM Motors S.p.A., which will be diagnosed by the suggested FDI scheme.

\section{Controlled turbocharged engine}

In this work, a diesel engine "Panther" RA428 equipped with a fixed geometry turbine, an external Exhaust Gas Recirculation (EGR) system, and a Throttle Valve Actuator (TVA) is considered, as shown in Fig. 1

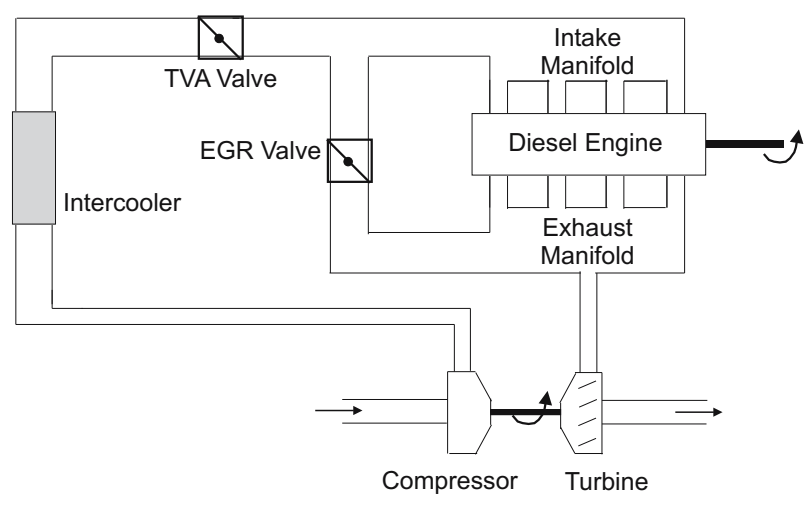

Fig. 1. Diesel engine air system.

This in-line 4 cylinder (2.8 1.) diesel motor is produced by VM Motors S.p.A. (Cento, Ferrara, Italy), but its engine is used in JK Jeep Wrangler outside of the US market. This engine main features are

- 2776 cc of displacement,

- 4 valve/per cylinder,

- Double Over Head Camshaft (DOHC),

- BOSCH common rail direct injection with electric piezo injectors operating at 30,000 psi,

- weight of 451 pounds / $205 \mathrm{~kg}$, power rating of 174 horsepower, 340 foot pounds.

In the following, the basics of the diesel engine physical model are briefly discussed, since its equations can be easily found in the literature (Pulkrabek, 2003).

For the 4 cylinder turbocharged diesel engine under investigation, it is assumed that the working 
fluid is a mixture of ideal gases always in equilibrium for all chemical compositions and pressure-temperature conditions. In order to simplify the fluid dynamics description, a dynamic model relying on the filling and emptying principle is set up (Pulkrabek, 2003). Assuming sufficiently small pipe dimensions, a lumped capacity representation is adopted, in which the fluid thermodynamic properties are spatially constant but time varying. In particular, this work considers an engine that is described by means of five elements: the turbine and the compressor, the intake and exhaust manifolds, and the cylinders. Each component is characterised by a different set of thermodynamic state variables and may be described by the ideal gas law, conservation of the mass, conservation of energy, and dynamic equilibrium equations.

In this paper, the real system described above is considered for the development and validation of the FDI scheme. To this aim, suitable residual generators have to be derived for the process under diagnosis, in their input-output forms. It is worth noting that sometimes the straightforward application of linear model-based fault diagnosis techniques can be difficult, due to dynamic model complexity. Thus, a viable procedure for practical application of FDI design techniques is truly necessary in real cases. Therefore, this work suggests to use fuzzy model identification for finding a solution of the fault diagnosis problem. In this way, complex physical or thermodynamic models are avoided. Moreover, the proposed TS fuzzy prototypes are used for the design of the fault diagnosis strategy. This is considered important to avoid the complexity that would otherwise be inevitable if purely nonlinear models were used.

Note that, in general, model-based fault diagnosis strategies require an accurate dynamic model of the diesel engine. This model can be derived via a "grey-box" modelling approach, which is based on the description of the input-output behaviour of the diesel engine from the first principle, i.e., starting directly at the level of established laws of physics. Also the parameters of the physical laws have to be empirically estimated. Both steps are complex and time consuming (for example, several months on a test bed). The high value variability of engine parameters makes, in the ideal case, an individual modelling for each produced engine necessary. Sometimes such parameter estimation cannot be applied to standard engines. Moreover, these estimation algorithms cannot be nowadays supported by the standard ECU (Electronic Control Unit) in terms of calculation power. Therefore, in order to improve diesel engine fault diagnosis, a strategy taking into account the engine characteristics, without an internal model, and with the calculation need should be provided. To meet these requirements, an off-line methodology relying on fuzzy identification of residual generators for fault diagnosis scheme design is thus proposed in this paper, as shown in Section 4

Finally, it is worth observing that the proposed approach, shown in Section 4 and relying on identified fuzzy generators, is not time consuming. Moreover, the optimisation stages required by both the estimation methods and the optimal threshold selection procedure described in Section 5] are performed off-line, using common computers with standard computation capabilities. However, the identified fuzzy prototypes for residual generation can be easily simulated on-line, once the optimisation stages have been performed off-line, since they are equivalent to look-up tables, as highlighted, e.g, by Rovatti et al. (2000), and thus easily supported by the standard ECU developed by BOSCH.

3.1. Fault mode and effect analysis. Various fault conditions have been simulated using the real diesel engine described here, which was developed by VM Motors S.p.A. In particular, VM Motors were interested in analysing three possible fault cases, which are considered in the following. They have been generated for testing the proposed FDI strategy and implemented via real-time rapid prototyping tools described in Section 6.2

In particular, the fault case 1 affects the turbocompressor behaviour, which is represented by the fouling of the surfaces of the compressor blades. It causes reduction in the air flow, changing the blade aerodynamics, and consequently varying the surface roughness. The fault causes a gradual decrease in the mass flow rate for a given pressure ratio.

In general, fouling is caused by the adherence of particles to airfoils and annulus surfaces. Compressor fouling is due to the size, amount, and chemical nature of the aerosols in the inlet air flow, dust, insects, organic matter such as seeds from trees, rust or scale from the inlet ductwork, carryover from coolers, or oil from leaky compressor bearing seals. Fouling must be distinguished from erosion, the abrasive removal of material from the flow path by hard particles impinging on flow surfaces. Erosion is probably more a problem for aero engine applications, because filtration systems used for automotive applications typically eliminates the bulk of the larger particles.

On the other hand, the fault case 2 describes the malfunctioning of the engine temperature thermocouple. This situation describes a temperature sensor fault, whose development rate is set to a certain percent error in the measured actual temperature, with respect to the time unit considered.

In general, this malfunction is due to a thermocouple decalibration process, which consists in unintentional alteration of the makeup of thermocouple wire. The usual cause is the diffusion of atmospheric particles into the metal at the extremes of operating temperature. Another 
cause is impurities and chemicals from the insulation diffusing into the thermocouple wire.

Finally, the fault case 3 affects the actuator of the TVA valve. This fault represents the loss of performance due to the wear of the TVA actuator. Under the assumption that there are no actuator dynamics, this fault causes a slower response of the TVA system. The actuator response time constant increases linearly with the time in order to represent a progressive damage to the actuator.

In general, many high-performance engines have throttle valves that are operated by an electric positioning motor. The throttle valve actuator can be sluggish since the electric motor may slowly wear out over time, causing it to operate more slowly than normal. This problem could be caused by electrical faults, since, for example, internal windings may have begun to fail, or the motor may be binding internally. On the other hand, mechanical ageing can mean bearing rust or a swelled rotor.

Note that, in realistic automotive applications, it is commonplace for each of the above faults to develop slowly over a period of months or years. For the purpose of this work, in order to avoid excessively long duration simulations, the fault development rate has been increased, so that significant effects are present after seconds. This factor must be taken into account in FDI algorithm design. On the other hand, the rate of development and magnitude of faults have been set to typical values. In fact, VM Motor S.p.A. were interested to know how small the fault parameters can be made while still maintaining good FDI performance. It is finally assumed that only a single fault may occur in the actuators, or the output sensor of the diesel engine.

The discussed fault modes that are of interest for VM Motors S.p.A. are modelled by means of ramp functions depicted in Figs. 24 As remarked above, these signals represent the case of incipient faults, i.e., hard to detect faults, thus modelled by means of ramp functions. As will be shown in Section 6 , their development rates (sizes versus lengths of time) have been suitably settled with respect to the corresponding measurement that these faults are affecting.

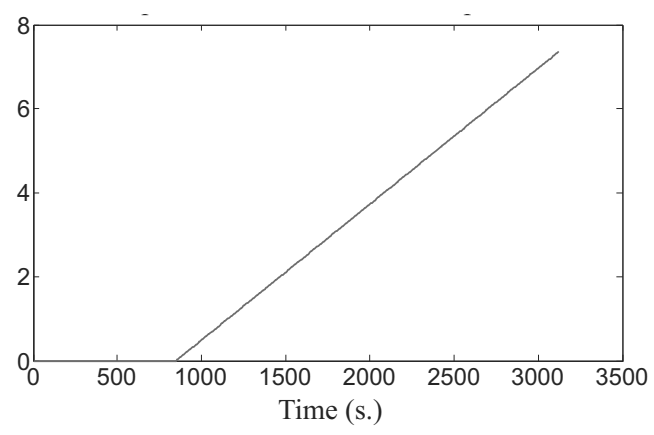

Fig. 2. Fault affecting the turbo-compressor

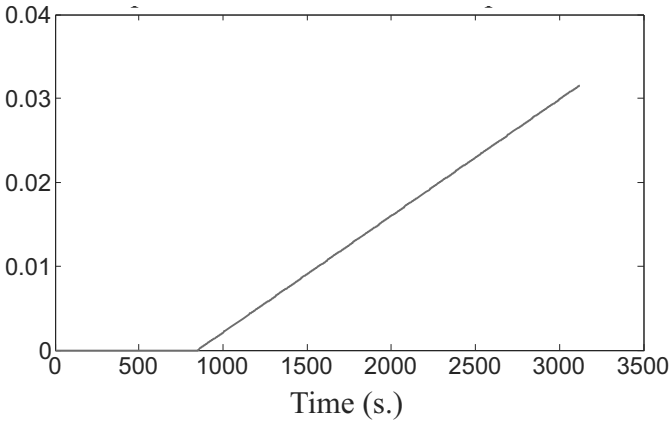

Fig. 3. Fault affecting the thermocouple sensor.

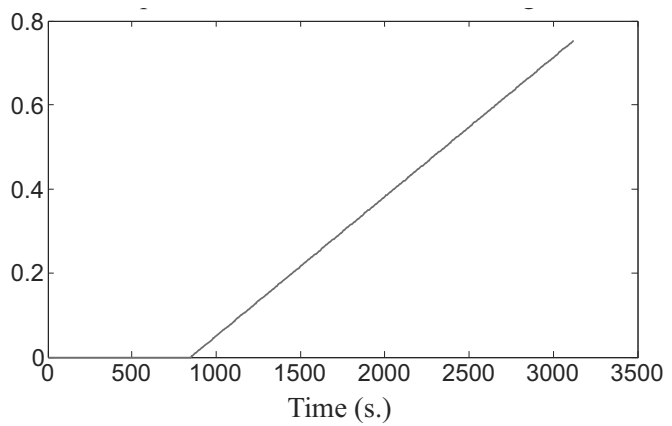

Fig. 4. Fault affecting the TVA control signal.

Moreover, Figs. 24 4 show that the fault development rates have been fixed in order to produce an error of about $5 \%$ per hour on the affected faulty engine measurements. As an example, the described faults commence at $850 \mathrm{~s}$.

The remainder of this section describes the relations among the fault cases considered above, and the monitored measurements acquired from the diesel engine. In this way, it will be shown that the fault isolation task can be easily solved. In particular, Table 1 shows fault effect distribution in the case of single fault occurrence, with respect to the acquired inputs $u(k)=$ $\left[u_{1}(k), \cdots, u_{6}(k)\right]$, and the output $y(k)$ (with $k=$ $1,2, \cdots, N)$ of the diesel engine.

Table 1. FMEA results for the diesel engine.

\begin{tabular}{|l|ccc|}
\hline Variable, measurement & \multicolumn{3}{|c|}{ Fault } \\
Case 1 & Case 2 & Case 3 \\
\hline \hline$u_{1}$, engine fuelling & 0 & 0 & 0 \\
$u_{2}$, engine speed & 0 & 0 & 0 \\
$u_{3}$, intake air flow temperature & 0 & 0 & 0 \\
$u_{4}$, engine temperature & 0 & 1 & 0 \\
$u_{5}$, EGR command & 0 & 0 & 0 \\
$u_{6}$, TVA command & 0 & 0 & 1 \\
$y$, intake air flow & 1 & 0 & 0 \\
\hline
\end{tabular}

Table 1 was obtained by performing the so-called fault sensitivity analysis, i.e., the Failure Mode \& Effect Analysis (FMEA) (Stamatis, 2003). In practice, Table 1 is 
thus obtained by selecting the most sensitive measurement ( $u_{i}$ or $y$ ) with respect to the simulated fault conditions (Case 1, Case 2, and Case 3). Obviously, when different fault conditions are considered with respect to the ones studied in this work, different measurements will probably have to be taken into account.

In particular, realistic fault signals, as depicted in Figs. 24, have been injected in the real diesel engine through the real-time tool described in Section 6.2, which represents also the well-known Hardware-In-Loop (HIL) simulation strategy.

More precisely, as described by (Stamatis, 2003), the process for conducting the FMEA can be based on a selection algorithm that is achieved here by introducing the normalised sensitivity function $N_{x}$ :

$$
N_{x}=\frac{S_{x}}{S_{x}^{*}}
$$

with

$$
S_{x}=\frac{\left\|\left.x(k)\right|_{f}-\left.x(k)\right|_{h}\right\|_{2}}{\left\|\left.x(k)\right|_{h}\right\|_{2}}
$$

and

$$
S_{x}^{*}=\max _{x} \frac{\left\|\left.x(k)\right|_{f}-\left.x(k)\right|_{h}\right\|_{2}}{\left\|\left.x(k)\right|_{h}\right\|_{2}} .
$$

It represents the effect of the fault case considered with respect to most sensitive input $u_{i}(k)$ or output $y(k)$, denoted in Eqn. (1) with the subscript $x$, i.e., $x(k)$. Thus, the function $S_{x}$ is defined by the ratio between the 2 -norm of the difference $\left.x(k)\right|_{f}-\left.x(k)\right|_{h}$ and the 2-norm of $\left.x(k)\right|_{h}$. The sequences $\left.x(k)\right|_{h}$ and $\left.x(k)\right|_{f}$ indicate the fault-free and faulty measurements $\left(u_{i}(k)\right.$ or $\left.y(t)\right)$, respectively.

Therefore, Table1 1 reports the measurements $u_{i}(k)$ or $y(k)$ that are mainly affected by the fault cases considered, which are denoted by ' 1 ' in the corresponding table entry. This situation corresponds to the case

$$
\max _{x} N_{x}=1 .
$$

On the other hand, an entry ' 0 ' means that the fault has smaller effects on the correspondent variable $x\left(u_{i}(k)\right.$ or $y(k))$, i.e.,

$$
\max _{x} N_{x}<1
$$

Table 1 is hence obtained with the evaluation of Eqn. (1). Thus, for each fault case, a different index $x$ satisfying Eqn. (1) determines the most sensitive signals $u_{i}(k)$ or $y(k)$.

Under these considerations, the entries ' 1 ' in Table 1 represent the variables $u_{i}(k)$ or $y(k)$ that are mainly sensitive to the fault case considered. It means that the sensitivity of the $x$-th measurement with respect to the fault signal considered is greater than any other different fault cases. On the other hand, the ' 0 ' entry means that the effect of the fault on the $i$-th output measurement can be neglected and therefore the fault considered does not affect the $x$-th output variable. The settlement of suitable thresholds for the evaluation of the relations of Eqns. (4) and (5) is not necessary, because of the normalisation with respect to the most sensitive fault effect $S_{x}^{*}$ in Eqn. (1).

Of course, it is worth noticing that the faults considered have barely detectable effects on any measurements, which represents the most challenging situation. On the other hand, these faults can be diagnosed using the strategy described in Sections 4 and 5 As an example, Fig. 5]depicts the engine measured temperature signal affected by a fault commencing at $850 \mathrm{~s}$, whose rate is fixed in order to produce an error of about $5 \%$ per hour on the corresponding temperature measurement $u_{4}(k)$.

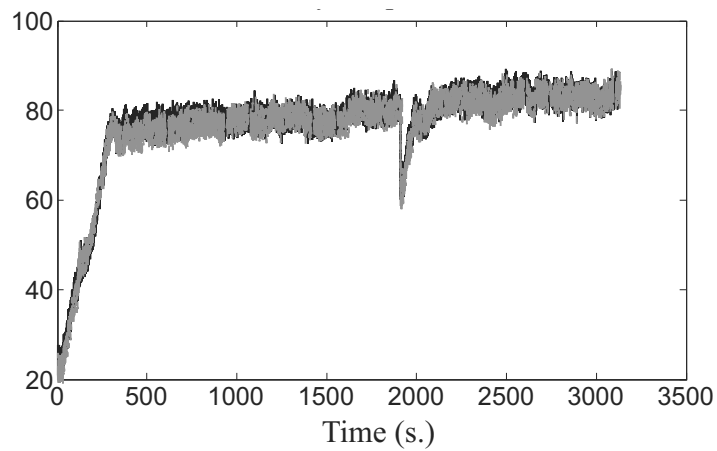

Fig. 5. Fault-free (continuous black) and faulty (dashed grey) engine temperature measurement $x=u_{4}(k)$.

From Fig. 5 it should be clear that any fault effect is not evident, but a fault diagnosis strategy must be exploited for detecting this fault effect. On the other hand, Fig. 6depicts the fault case 1 regarding the intake air flow measurement $x=y(k)$, whilst Fig. 7 reports the TVA actuator signal $x=u_{6}(k)$ for the fault case 2 .

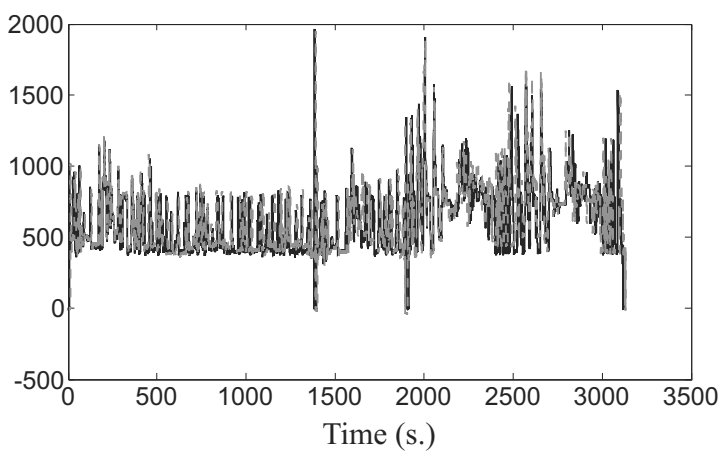

Fig. 6. Fault-free (continuous black) and faulty (dashed grey) intake air flow measurement $x=y(k)$.

Finally, it was assumed that only a single fault may occur in the actuators, components, or output sensors of 


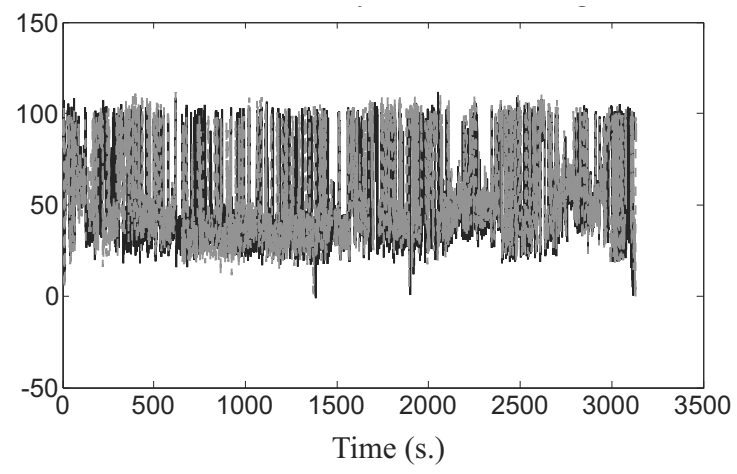

Fig. 7. Fault-free (continuous black) and faulty (dashed grey) TVA actuator signal $x=u_{6}(k)$.

the plant considered. However, on the basis of fault effect analysis, faults occurring at the same time can be distinguished by analysing their effects on the monitored measurements via fault sensitivity analysis. This analysis also simplifies the identification procedure, as it reduces the number of input and output measurements that have to be monitored by the proposed FDI scheme.

\section{Fuzzy modelling and identification}

This section addresses the approach exploited for obtaining the mathematical description of the residual generators applied to the diesel engine. In particular, the fuzzy identification scheme, which is recalled in Section 4.1 allows the design of the proposed fault diagnosis scheme shown in Section 5 .

4.1. Fuzzy identification from data. The approach suggested in this work relies on the identification of transparent rule-based fuzzy prototypes, which are used as residual generators for the the system under diagnosis. From the system identification point of view, a fuzzy model is regarded as a composition of local affine submodels, whilst fuzzy sets naturally provide smooth transitions between these submodels.

In order to generate fuzzy models automatically from measurements, a comprehensive methodology is used. This employs fuzzy clustering techniques to partition the available data into subsets characterised by a linear behaviour. The relationships between the presented identification method and linear regression are exploited, allowing for the combination of fuzzy logic techniques with system identification tools. In addition, an implementation in the Matlab ${ }^{\circledR}$ Toolbox of the Fuzzy Modelling and IDentification (FMID) technique presented in the following is available (Babuška, 2000). Fuzzy identification usually refers to techniques and algorithms for constructing fuzzy models from data.
In this section, fuzzy descriptions are viewed as a class of local modelling approaches, which attempt to solve a complex modelling problem by decomposing it into a number of simpler subproblems. In particular, fuzzy logic is exploited to define a TS fuzzy model (Takagi and Sugeno, 1985). The TS fuzzy model for nonlinear dynamic systems is described by a collection of local linear or affine submodels, each one approximating the system behaviour around a single working point. The TS fuzzy model is thus able to describe the global behaviour of the nonlinear system. The scheduling of the submodels is achieved through a smooth function of the system state, the behaviour of which is defined using fuzzy set theory (Babuška, 1998).

A large part of fuzzy modelling and identification algorithms (see, e.g., Simani et al., 1999) share a common two-step procedure, in which at first the operating regions are determined using heuristics or data clusterings techniques. Then, in the second stage, the identification of the parameters of each submodel is achieved using a suitable estimation algorithm. From this perspective, fuzzy identification can be regarded as a search for a decomposition of a nonlinear behaviour, which gives a desired balance between the complexity and the accuracy of the model, effectively exploring the fact that the complexity of systems is usually non-uniform. Since it cannot be expected that sufficient prior knowledge is available concerning this decomposition, methods for automated generation of the decomposition, primarily from system data, are developed. A suitable class of fuzzy clustering algorithms is used for this purpose.

\subsubsection{Takagi-Sugeno multiple-model paradigm.} The fuzzy rule-based model suitable for the approximation of a large class of nonlinear systems was introduced by Takagi and Sugeno (Takagi and Sugeno, 1985). In the TS fuzzy model, the rule consequents are crisp functions of the model inputs:

$$
R_{i}: \text { IF } \mathbf{x} \text { is } A_{i} \text { THEN } y_{i}=f_{i}(\mathbf{x}),
$$

where $i=1,2, \ldots, M, \mathbf{x} \in \mathbb{R}^{p}$ is the input (antecedent) variable and $y_{i} \in \mathbb{R}$ is the output (consequent) variable. $R_{i}$ denotes the $i$-th rule, and $M$ is the number of rules in the rule base. $A_{i}$ is the antecedent fuzzy set of the $i$-th rule, defined by a (multivariate) membership function.

The consequent functions $f_{i}$ are typically chosen as instances of a suitably parameterised function, whose structure remains equal in all the rules and only the parameters vary. A simple and practically useful parameterisation of the function $f_{i}$ is the affine form,

$$
y_{i}=\mathbf{a}_{i} \mathbf{x}+b_{i},
$$

where $\mathbf{a}_{i}$ is the parameter vector (regressand) and $b_{i}$ is the scalar offset. $\mathbf{x}=\mathbf{x}(k)$ represents the regressor vector, 
which can contain delayed samples of $u(k)$ and $y(k)$. This model is referred to as the affine TS model, and can be written as (Takagi and Sugeno, 1985)

$$
y=\frac{\sum_{i=1}^{M} \mu_{i}(\mathbf{x}) y_{i}}{\sum_{i=1}^{M} \mu_{i}(\mathbf{x})} .
$$

The antecedent fuzzy sets $\mu_{i}$ are extracted from the fuzzy partition matrix (Babuška, 1998). The consequent parameters $\mathbf{a}_{i}$ and $b_{i}$ are estimated from the data using the method developed by the author (Simani et al., 1999) and recalled below. This identification scheme exploited for the estimation of TS model parameters has been integrated into the FMID toolbox for Matlab ${ }^{\circledR}$ by the author. This approach developed by the author is usually preferred when the TS model should serve as a predictor, as it computes the consequent parameters via the Frisch scheme, developed for errors-in-variables descriptions (Van Huffel and Lemmerling, 2002). Therefore, after the clustering of the data has been obtained via the GK algorithm (Babuška, 1998), the data subsets are processed according to the Frisch scheme identification procedure (Simani et al., 1999), in order to estimate the TS parameters for each affine submodel.

4.1.2. Fuzzy identification from clusters. As stated above, the GK fuzzy clustering algorithm is used to approximate a data set by local affine models. In order to obtain a description useful for prediction purposes, an additional step must be applied to generate models independent of the identification data. This section recalls the algorithm for constructing fuzzy TS prototypes from fuzzy partitions.

The antecedent fuzzy sets $A_{i}$ can be computed analytically in the antecedent product space or extracted from the fuzzy partition matrix. The consequent parameters $\mathbf{a}_{i}$ and $b_{i}$ are estimated from the data using the method sketched in the following. The antecedent membership functions can be obtained by projecting the fuzzy partition onto the antecedent variables or computing the membership degrees directly in the product space of the antecedent variables. These methods are available from the FMID toolbox for Matlab ${ }^{\circledR}$ (Babuška, 2000) developed by Robert Babuška (Babuška, 1998). The method exploited in this study is the second one, which considers multi-dimensional antecedent membership functions, represented analytically by computing an inverse of the distance from the cluster prototype. The membership degree is computed directly for the entire input vector (without the decomposition). The antecedents of the TS rules are simple propositions with multi-dimensional fuzzy sets $\mu(\mathbf{x})$ of Eqn. (8).

Regarding the estimation of the consequent parameters, they are derived using the procedure recalled in the following and developed by the author and his co-workers (Simani et al., 1999). This approach is preferred when TS descriptions should serve as predictors.

Thus, after the clustering of the data has been obtained, in order to identify the structure of the TS prototype of Eqn. (8) in the $i$-th cluster, with $i=$ $1, \ldots, K$, and $K$ clusters, the following matrices are defined:

$$
X_{n}^{(i)}=\left[\begin{array}{ccc}
y(k) & \mathbf{x}_{n}^{T}(0) & 1 \\
y(k+1) & \mathbf{x}_{n}^{T}(1) & 1 \\
\vdots & \vdots & \vdots \\
y\left(k+N_{i}-1\right) & \mathbf{x}_{n}^{T}\left(N_{i}-1\right) & 1
\end{array}\right],
$$

where the subscript $n$ represents the order of the dynamic model considered (number of regressors), i.e., $\mathbf{x}_{n}(h)=$ $[y(h-1), \ldots, y(h-n), u(h-1), \ldots, u(h-n)]^{T}$. Therefore

$$
\Sigma_{n}^{(i)}=\left(X_{n}^{(i)}\right)^{T} X_{n}^{(i)} .
$$

In order to solve the so-called noise-rejection problem (Simani et al., 1999) in a mathematical framework, it is necessary to follow the assumptions that the noises $\tilde{u}(k)$ and $\tilde{y}(k)$ are additive on the input-output data $u^{*}(k)$ and $y^{*}(k)$, and region independent $(k=1,2, \ldots, N)$.

Under these assumptions, a positive-definite matrix $\Sigma_{n}^{(i)}$ associated to the sequences belonging to the $i$-th cluster can be expressed as the sum of two terms $\Sigma_{n}^{(i)}=$ $\Sigma_{n}^{*(i)}+\overline{\tilde{\Sigma}}_{n}$, where

$$
\overline{\tilde{\Sigma}}_{n}=\operatorname{diag}\left[\overline{\tilde{\sigma}}_{y} I_{n+1}, \overline{\tilde{\sigma}}_{u} I_{n}, 0\right] \geq 0 .
$$

The solution of the above identification problem requires the computation of the unknown noise covariances $\overline{\tilde{\sigma}}_{u}$ and $\overline{\tilde{\sigma}}_{y}$, which can be achieved by solving the relation

$$
\Sigma_{n}^{*(i)}=\Sigma_{n}^{(i)}-\tilde{\Sigma}_{n} \geq 0
$$

in the variables $\tilde{\sigma}_{u}, \tilde{\sigma}_{y}$, where

$$
\tilde{\Sigma}_{n}=\operatorname{diag}\left[\tilde{\sigma}_{y} I_{n+1}, \tilde{\sigma}_{u} I_{n}, 0\right] .
$$

It is worth noting that all the surfaces defined by Eqn. (12 have necessarily at least one common point, i.e., the point $\left(\overline{\tilde{\sigma}}_{u}, \overline{\tilde{\sigma}}_{y}\right)$ corresponding to the true variances of the noise affecting the input and the output data.

The search for a solution to the identification problem can therefore start from the determination of this point in the noise space, if the noise characteristics are common to all the clusters and all assumptions regarding the Frisch scheme are satisfied (independence between input-output sequences, additive noise, noise whiteness) (Van Huffel and Lemmerling, 2002).

However, in real cases, these assumptions have to be relaxed. Thus no common point can be determined among surfaces $\Gamma_{n}^{(i)}=0$ (i.e., the locus of the points 
satisfying Eqn. (12) in the noise plane, and a unique solution to the identification problem cannot be obtained. In this situation, local fuzzy model identification can be performed by finding a point $\left(\tilde{\sigma}_{u}, \tilde{\sigma}_{y}\right) \in \Gamma_{n+1}^{(i)}=0$ that makes $\Sigma_{n+1}^{*(i)}$ closer to the double singular condition. It leads to determining the common point of the surfaces even when the assumptions of the Frisch scheme are slightly violated. Moreover, for each $i$-th cluster, different noises $\left(\overline{\tilde{\sigma}}_{u}^{(i)}, \overline{\tilde{\sigma}}_{y}^{(i)}\right)$ and the following relation should be rewritten as

$$
\Sigma_{n}^{*(i)}=\Sigma_{n}^{(i)}-\tilde{\Sigma}_{n}^{(i)} \geq 0
$$

where $\tilde{\Sigma}_{n}^{(i)}=\operatorname{diag}\left[\overline{\tilde{\sigma}}_{u}^{(i)} I_{n+1}, \overline{\tilde{\sigma}}_{y}^{(i)} I_{n}, 0\right]$ whilst $\left(\overline{\tilde{\sigma}}_{u}^{(i)}, \overline{\tilde{\sigma}}_{y}^{(i)}\right)$ represent the variances of input and output additive noises in the $i$-th cluster. The identification scheme considered normally assumes that (Van Huffel and Lemmerling, 2002)

$$
\left\{\begin{array}{l}
u(k)=u^{*}(k)+\tilde{u}(k), \\
y(k)=y^{*}(k)+\tilde{y}(k),
\end{array}\right.
$$

where $u^{*}(k)$ and $y^{*}(k)$ are the noise-free data noise terms $\tilde{u}(k)$ and $\tilde{y}(k)$ are independent of every other term, and only $u(k)$ and $y(k)$ are known.

Finally, the matrices $\tilde{\Sigma}_{n}^{(i)}$ can therefore be built and the parameter of the model in each cluster can be determined by means of the relation:

$$
\left(\Sigma_{n}^{(i)}-\tilde{\Sigma}_{n}^{(i)}\right) \mathbf{a}^{(i)}=\mathbf{0}, \quad i=1, \ldots, K,
$$

for $K$ clusters. This completes the fuzzy identification procedure in the fuzzy environment.

Finally, on the basis of the results achieved here, Section 5 will describe the design of residual generators in the form of fuzzy TS prototypes of Eqn. (8) for the FDI of the diesel engine considered.

\section{Fault diagnosis scheme design}

This section addresses the problem of the detection and isolation of faults affecting the process under diagnosis. In the following, it is assumed that the monitored system in terms of input-output signals has the structure depicted in Fig. 8. The term $y(k)$ is the system output measurement, and $u(k)$ the control input.

According to the expressions of Eqn. 114, in realistic situations the variables $u^{*}(k)$ and $y^{*}(k)$ are measured by means of sensors, whose outputs are affected by noise.

Neglecting sensor dynamics, faults affecting the measured input and output signals $u(k)$ and $y(k)$ are modelled as

$$
\left\{\begin{array}{l}
u(k)=u^{*}(k)+f_{u}(k), \\
y(k)=y^{*}(k)+f_{y}(k),
\end{array}\right.
$$

Input Measurements

Output Measurements

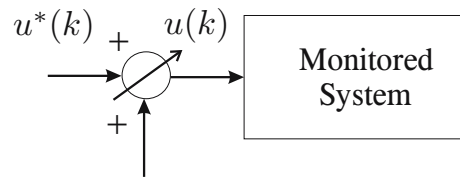

$f_{u}(k)$

Actuator Faults

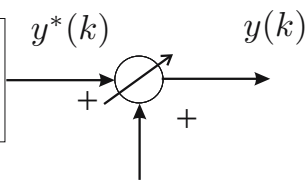

$f_{y}(k)$
Fig. 8. Structure of the monitored system.

where $f_{u}(k)$ and $f_{y}(k)$ represent additive signals, which assume values different from zero only in the presence of faults.

There are different approaches to generate diagnostic signals, i.e., residuals (or symptoms), from which it will be possible to diagnose the fault cases considered. In this work, TS fuzzy prototypes are used as residual generators. As depicted in Fig. 9, the residual signals are generated by the comparison of the measured $y(k)$ and the estimated $\hat{y}(k)$ output.

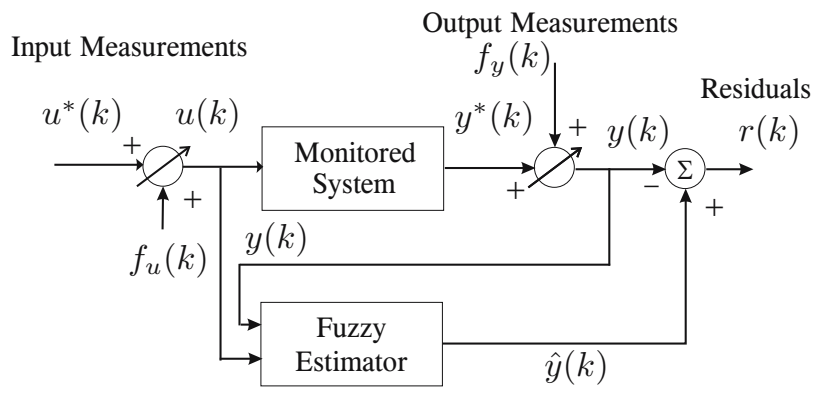

Fig. 9. Residual generation scheme exploiting TS prototypes.

Residual evaluation refers to a logic device which processes the redundant signals generated by the first block in order to detect when a fault occurs, and to univocally identify the unreliable actuator or sensor.

The fault detection task is performed by using a simple thresholding logic of Eqn. (17),

$$
\left\{\begin{array}{l}
\bar{r}-\delta \sigma_{r} \leq r(k) \leq \bar{r}+\delta \sigma_{r} \\
\text { if fault-free } \\
r(k)<\bar{r}-\delta \sigma_{r} \text { or } r(k)>\bar{r}+\delta \sigma_{r} \\
\text { if faulty. }
\end{array}\right.
$$

It is worth noting that the incipient faults considered in this paper, and described in Section 3.1. which are modelled by ramp functions, may not be immediately detected, since the delay in the corresponding alarm normally depends on the fault mode. This situation is 
shown in Fig. 10, where the fault detection thresholds are computed according to the expression of Eqn. (17).

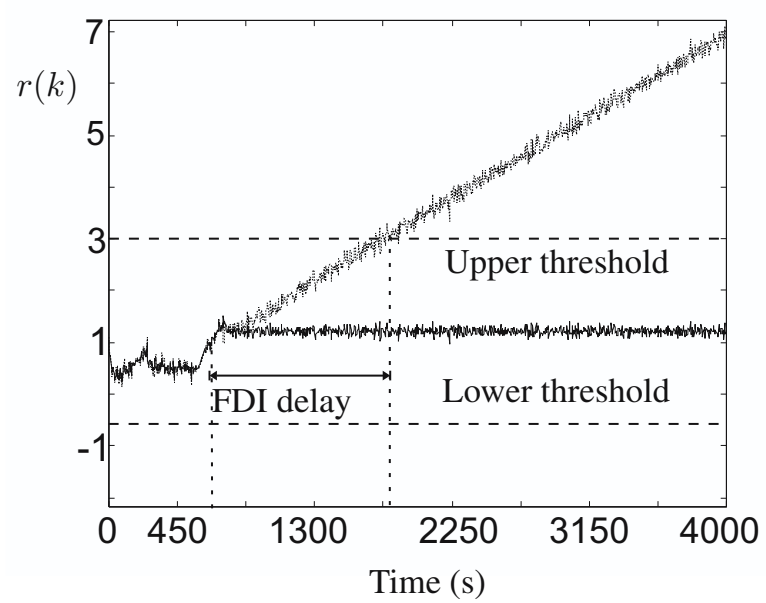

Fig. 10. Detection thresholds and delay for incipient faults.

In practice, the residual signal is represented by the stochastic variable $r(k)$, whose sample mean and variance values are estimated as follows:

$$
\left\{\begin{array}{l}
\bar{r}=\frac{1}{N} \sum_{k=1}^{N} r(k), \\
\sigma_{r}^{2}=\frac{1}{N} \sum_{k=1}^{N}[r(k)-\bar{r}]^{2},
\end{array}\right.
$$

where $\bar{r}$ and $\sigma_{r}^{2}$ are the values for the sample mean and variance of the fault-free residual, respectively. $N$ is the number of samples of the signal $r(k)$. The values of $\bar{r}$ and $\sigma_{r}^{2}$ depend on the signal $r(k)$ statistics, which are usually unknown.

In order to separate the normal behaviour from a faulty, a tolerance parameter $\delta$ (normally $\delta \geq 2$ ) is selected and properly tuned. Hence, by a proper choice of this parameter $\delta$, a good trade-off can be achieved between the maximisation of the fault detection probability and the minimisation of the false alarm rate. This parameter $\delta$ could be fixed with empirical rules or, once the values of $\bar{r}$ and $\sigma_{r}^{2}$ have been estimated from the $r(k)$ signal, using the three-sigma rule. On the other hand, less conservative results could be obtained exploiting a procedure borrowed from the aerospace framework, which leads to determining via extensive simulations the optimal $\delta$ minimising the false alarm rate and maximising the detection/isolation probability. This issue will be addressed, along with the achieved results, in Section 6 .

Therefore, the minimal detectable fault can be found by fixing a proper parameter $\delta$, as shown in Fig. 10. If a detection delay is tolerable, which depends on fault severity, the amplitude of the minimal detectable fault can be lower. In this case, the minimal detectable faults on various sensors depend on industrial and automotive applications, by considering also that the minimal detectable faults can be reduced if a delay in detection promptness is tolerable.

Finally, regarding the fault isolation problem, a "generalised estimator scheme" has to be exploited. In particular, as shown in Section 3.1, since only one fault $f_{y}(k)$ affects the output measurement $y(k)$, whilst two faults $f_{u}(k)$ regard the input measurements $u_{4}(k)$ and $u_{6}(k)$, to uniquely isolate the fault $f_{u}(k)$ concerning one of the inputs $u_{i}(k)$, under the assumption that the output $y(k)$ is fault-free, a bank of fuzzy estimators in the form of Eqn. (8) is used, as shown in Fig. 11

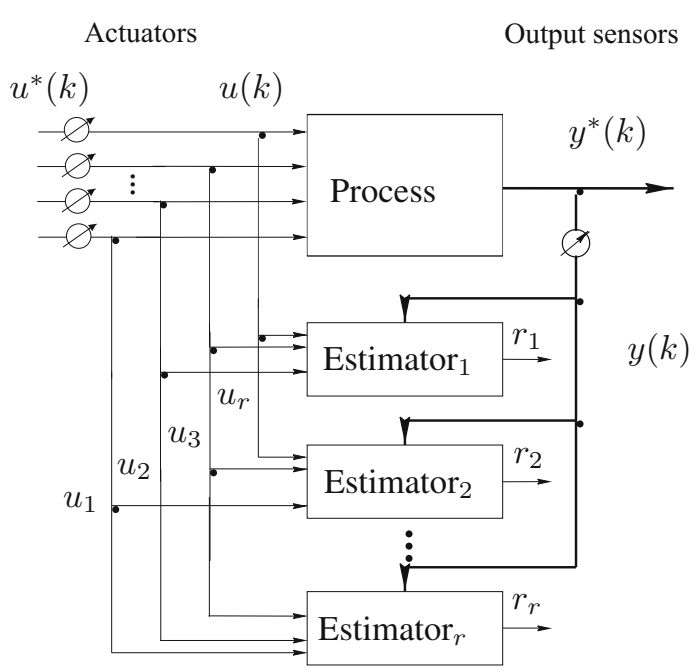

Fig. 11. Fuzzy estimator scheme for fault isolation.

The number of these fuzzy estimators is equal to that of fault $f_{u}(k)$ that has to be diagnosed. The $i$-th fuzzy estimator is driven by all but the $i$-th input $u_{i}(k)$ (or even more inputs, if necessary) and the system output, and generates a residual function which is sensitive to all but the $i$-th input fault $f_{u}(k)$ (or even more inputs, if necessary). The identification procedure for these output fuzzy estimators follows that described in Section 4. In particular, when the fuzzy estimator insensitive to the $i$-th input (or even more inputs, if necessary) has to be designed, the output $y(k)$ and all but the $i$-th inputs $u_{i}(k)$ are exploited for the identification process. In this way, fault isolation is possible, since a fault on the $i$-th input affects all the residual functions except that of the device which is insensitive to the $i$-th input, i.e., $r_{i}$, or even more inputs, if required.

The effectiveness of the identifiability of fuzzy residual generators as well as the capabilities of fuzzy fuzzy prototypes from all inputs except one (or more) are assessed in Section 6. Moreover, the properties of fuzzy prototypes as universal approximators, and the 
identification capabilities of fuzzy output predictors have been investigated, e.g., by Simani et al. (2003), Rovatti (1996), or Fantuzzi and Rovatti (1996).

In order to summarise the isolation capabilities of the presented schemes, Table 2 shows the so-called fault signature for the case of a single fault in each input signal.

Table 2. Fault signatures

\begin{tabular}{|c|cccc|}
\hline & $u_{1}$ & $u_{2}$ & $\ldots$ & $u_{r}$ \\
\hline \hline$r_{1}$ & 0 & 1 & $\ldots$ & 1 \\
$r_{2}$ & 1 & 0 & $\ldots$ & 1 \\
$\vdots$ & $\vdots$ & $\vdots$ & $\vdots$ & $\vdots$ \\
$r_{r}$ & 1 & 1 & $\ldots$ & 0 \\
\hline
\end{tabular}

The residuals which are affected by input faults are described by an entry ' 1 ' in the corresponding table entry, while an entry ' 0 ' means that the fault considered does not affect the corresponding residual. Note that, with reference to the present study, it is not necessary to isolate faults in the system output $y(k)$ since it is assumed that only one single fault can occur on the process output, which is a scalar signal $y(k)$.

This work does not consider the fault estimation problem, which was addressed in other works by the same author ((Bonfè et al., 2011) but using a different approach. However, fuzzy TS models, which are used here as residual generators, could be exploited for fault signal reconstruction, as shown, e.g., by Xu et al. (2012), in the same way as for neural networks (Korbicz et al., 2004).

Finally, it is worth noticing that the identified fuzzy prototypes for residual generation can be easily simulated on-line, once the optimisation stages have been performed off-line, since they are equivalent to look-up tables and thus easily implementable by standard ECUs.

\section{Experimental results}

This section describes experimentations with the method proposed for the fuzzy identification technique oriented to the design of fuzzy residual generators used for diesel engine fault diagnosis.

6.1. Diesel engine modelling validation. The fuzzy identification procedure recalled in Section 4 exploits the design of fault diagnosis residual generators based on identified TS fuzzy prototypes. In particular, once the input-output data have been acquired from the real diesel engine process, both residual generator parameter identification and fuzzy clustering tasks have been performed off-line.

It is assumed that the monitored diesel engine, depicted in Fig. 1, normally works in nominal fault-free conditions. In general, the process operates in different working conditions, and the seven input-output measurements $u(k)$ and $y(k)$, including temperatures, flows, control signals, and speed, can be acquired with a sampling rate $T_{s}=0.1 \mathrm{~s}$.

More precisely, the acquired inputs $u(k)=$ $\left[u_{1}(k), \ldots, u_{6}(k)\right]$ and the output $y(k)$ (with $k=$ $1,2, \ldots, N)$ of the diesel engine are explained in Table 3

Table 3. Process inputs and output

\begin{tabular}{|c|l|}
\hline Signals & Measurements \\
\hline \hline$u_{1}$ & engine fuelling \\
$u_{2}$ & engine speed \\
$u_{3}$ & intake air flow temperature \\
$u_{4}$ & engine oil temperature \\
$u_{5}$ & EGR command \\
$u_{6}$ & TVA command \\
$y$ & intake air flow \\
\hline
\end{tabular}

On the other hand, Table 4 reports the measurement errors of the input-output signals acquired from the real diesel engine test-rig, and expressed as a percent of normalised standard deviations of the corresponding measurements. They were empirically estimated taking into account the sensing devices performing the measurements, as well as the nominal accuracy guaranteed by the ECU developed by BOSCH.

Table 4. Input and output signal accuracy.

\begin{tabular}{|l|c|}
\hline Measurement & Accuracy \\
\hline \hline engine fuelling & $7 \%$ \\
engine speed & $4 \%$ \\
intake air flow temperature & $2 \%$ \\
engine oil temperature & $2 \%$ \\
EGR command signal & $6 \%$ \\
TVA command signal & $4 \%$ \\
intake air flow & $4 \%$ \\
\hline
\end{tabular}

On the other hand, the clustering algorithm recalled in Section 4.1.1 was implemented off-line and provided the optimal number of clusters (operating conditions), as well as the number of sample delays of the inputs and outputs for models of Eqn. (8). After clustering, the parameters $\mathbf{a}_{i}$ and $b_{i}$, with $i=1, \ldots, K$ and $j=$ $1, \ldots, n$, were estimated off-line using the proposed identification scheme. Moreover, as suggested at the end of Section 5 in order to achieve the fault isolation task, three fuzzy estimators in the form of Eqn. (8) are considered.

It is worth noticing that the measurement noise error variance values $\left(\overline{\tilde{\sigma}}_{u}, \overline{\tilde{\sigma}}_{y}\right)$ estimated according to the procedure recalled in Section 4.1.2 and reported in Table 5] seem to be consistent with the actual measurement accuracy values summarised in Table 4 
Table 5. Estimated measurement noise variance values $\left(\overline{\tilde{\sigma}}_{u}, \overline{\tilde{\sigma}}_{y}\right)$.

\begin{tabular}{|l|c|}
\hline Measurement & Accuracy \\
\hline \hline engine fuelling & $7.06 \%$ \\
engine speed & $3.99 \%$ \\
intake air flow temperature & $1.81 \%$ \\
engine oil temperature & $1.93 \%$ \\
EGR command signal & $5.78 \%$ \\
TVA command signal & $3.82 \%$ \\
intake air flow & $3.96 \%$ \\
\hline
\end{tabular}

According to Figs. 9 and 11 the required fault diagnosis residuals are generated by three TS fuzzy Multiple-Input Single-Output (MISO) prototypes of Eqn. (8). Thus, by following the scheme of Fig. 11, one fuzzy predictor used for the computation of the residual $r_{1}(k)$ is fed by the output $y(k)$ and four inputs $\left[u_{1}(k), u_{2}(k), u_{3}(k), u_{5}(k)\right]$ with $K=$ 7 and $n=3$. The second fuzzy estimator generating $r_{3}(k)$ is fed by $y(k)$ and five inputs, e.g., $\left[u_{1}(k), u_{2}(k), u_{3}(k), u_{5}(k), u_{6}(k)\right]$ with $K=7$ and $n=3$. Finally, the third fuzzy estimator for $r_{2}(k)$ is fed by $y(k)$ and $\left[u_{1}(k), u_{2}(k), u_{3}(k), u_{4}(k), u_{5}(k)\right]$ with $K=7$ and $n=3$. The membership degrees $\mu_{i}$ required by the fuzzy estimators of Eqn. (8) have been approximated with Gaussian functions, whose parameters have been estimated by the fuzzy clustering algorithm (Babuška, 2000).

Therefore, the complete fuzzy estimator strategy is obtained by following Table 1, as these estimators, organised into a bank structure, after fault detection allow performing also the required fault isolation task, as described in Section 5 .

It is worth noticing that the identified fuzzy prototypes for residual generation have been easily simulated on-line, once the optimisation stages have been performed off-line. The computation time required for the simulation of fuzzy TS models is quite low, thus allowing, if necessary, real-time generation of the residual signals $r(k)$. However, the computation time required for both model estimation and optimisation is not crucial here since these tasks are performed off-line at the FDI scheme design stage.

As an example, Figs. 12 17 depict one of the data sets containing the inputs signals $u(k)$ generated from the process under diagnosis.

Note that the range limitations of the $u_{5}(k)$ and $u_{6}(k)$ signals depicted in Figs. 16 and 17 are due to the control strategies implemented by the ECU BOSCH controller used by VM Motors S.p.A.

On the other hand, the actual output measurement, $y(k)$, compared with the signal reconstructed by the fuzzy estimator, is reported in Fig. 18

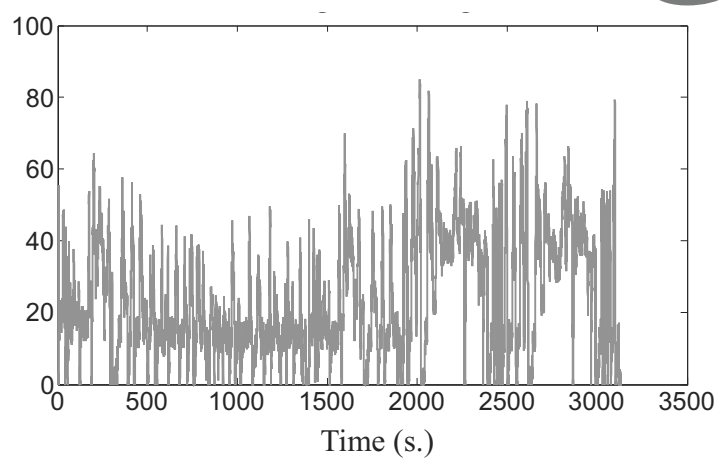

Fig. 12. Actual input $u_{1}(k)$ from the monitored process.

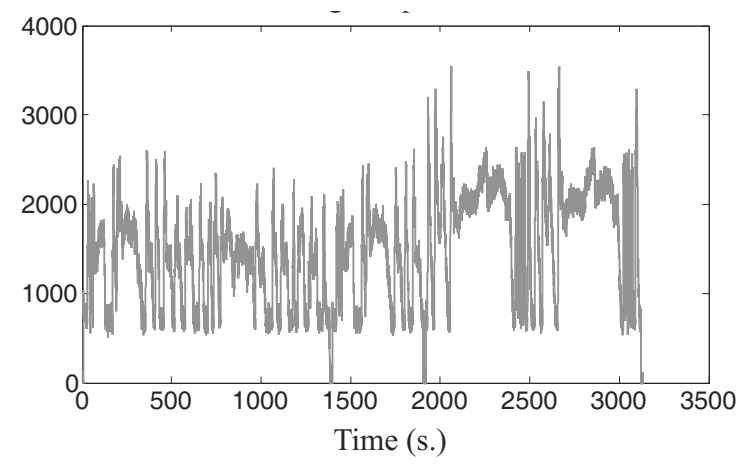

Fig. 13. Actual input $u_{2}(k)$ from the monitored process.

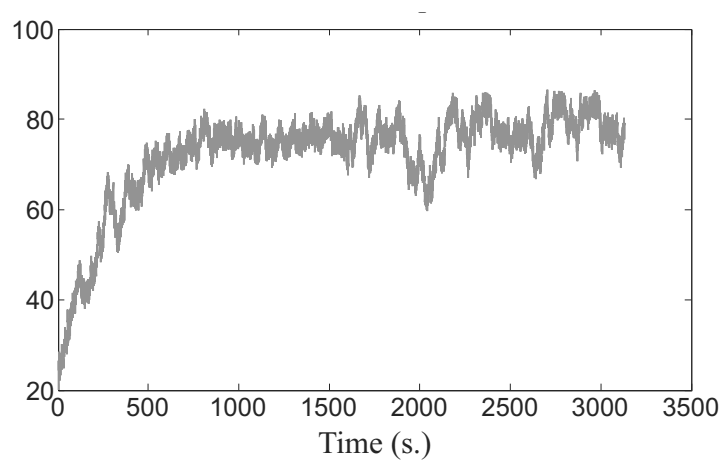

Fig. 14. Actual input $u_{3}(k)$ from the monitored process.

In order to highlight the performance of TS fuzzy models, Fig. 19 represent the zoom of the actual output measurement, $y(k)$, compared with the signal reconstructed by the fuzzy estimator for $r_{1}(k)$ residual generation.

In the following, the structure of fuzzy residual generators is reported. In particular, regarding the generation of the residual $r_{1}(k)$ on the basis of the signals $u_{1}(k), u_{2}(k), u_{3}(k), u_{5}(k)$, and $y(k)$, the identified consequents are reported in the following expressions for 


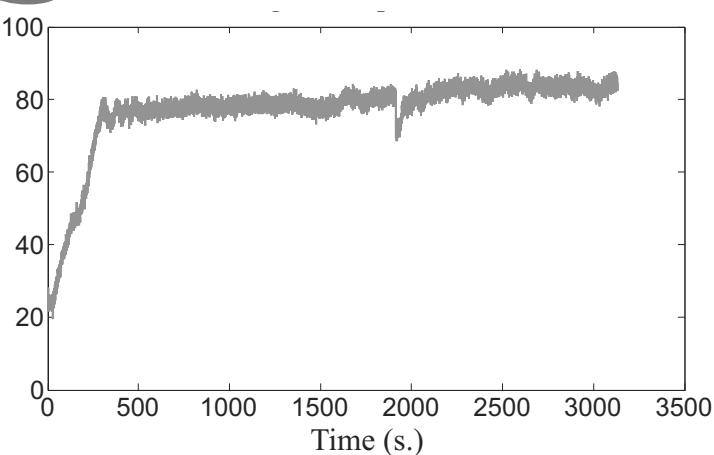

Fig. 15. Actual input $u_{4}(k)$ from the monitored process.

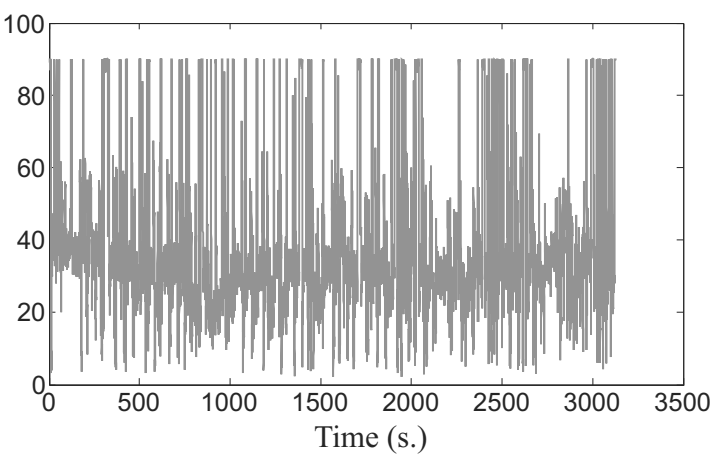

Fig. 16. Actual input $u_{5}(k)$ from the monitored process.

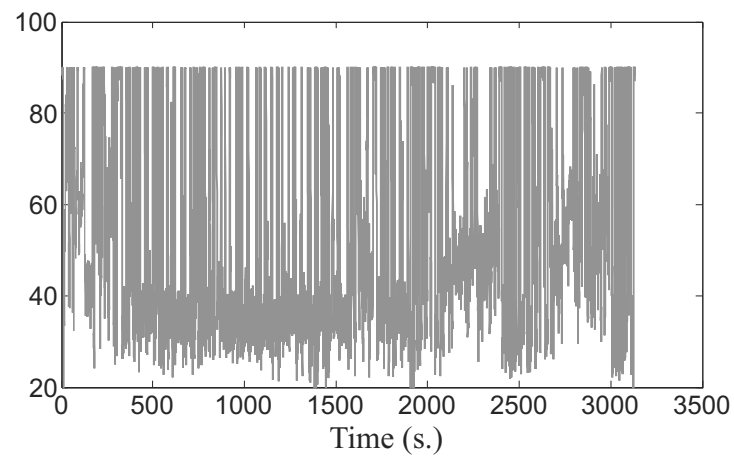

Fig. 17. Actual input $u_{6}(k)$ from the monitored process.

each rule:

Rule 1:

$r_{1}(k)$

$$
\begin{aligned}
= & 0.97 y(k-1)-8.93 \cdot 10^{-4} y(k-2) \\
& +4.01 \cdot 10^{-2} y(k-3)+9.12 u_{1}(k-1) \\
& -11.6 u_{1}(k-2)+2.46 u_{1}(k-3) \\
& -0.121 u_{2}(k-1)+0.34 u_{2}(k-2) \\
& -0.21 u_{2}(k-3)+4.28 u_{3}(k-1) \\
& -0.423 u_{3}(k-2)-3.77 u_{3}(k-3) \\
& -0.61 u_{5}(k-1)+0.47 u_{5}(k-2) \\
& +0.13 u_{5}(k-3)-16-y(k),
\end{aligned}
$$

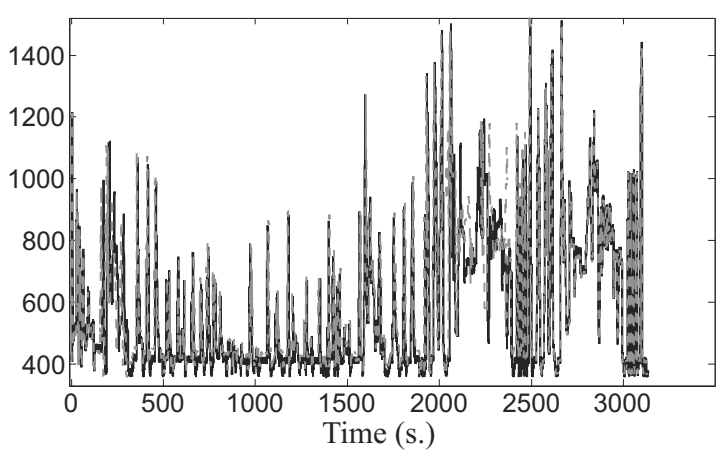

Fig. 18. Simulated (dashed grey) and real (continuous black) process output (intake air flow).

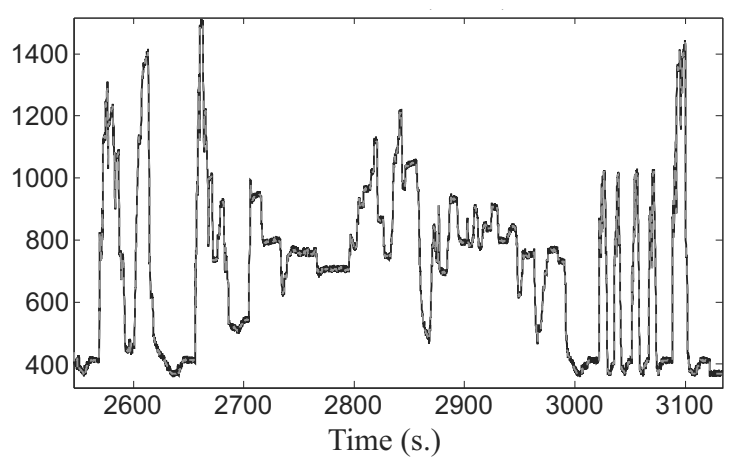

Fig. 19. Zoom of the simulated (dashed grey) and real (continuous black) process output.

Rule 2:

$$
\begin{aligned}
& r_{1}(k) \\
& =1.04 y(k-1)-3.41 \cdot 10^{-2} y(k-2) \\
& \quad-1.21 \cdot 10^{-4} y(k-3)-0.23 u_{1}(k-1) \\
& \quad+0.32 u_{1}(k-2)-0.17 u_{1}(k-3) \\
& \quad-1.17 \cdot 10^{-2} u_{2}(k-1) \\
& \quad-2.28 \cdot 10^{-3} u_{2}(k-2)+1.24 \cdot 10^{-2} u_{2}(k-3) \\
& \quad-0.62 u_{3}(k-1)+0.12 u_{3}(k-2) \\
& \quad+0.54 u_{3}(k-3)-1.60 \cdot 10^{-2} u_{5}(k-1) \\
& \quad+7.64 \cdot 10^{-2} u_{5}(k-2) \\
& \quad-7.20 \cdot 10^{-2} u_{5}(k-3)+1-y(k),
\end{aligned}
$$

Rule 3:

$$
\begin{aligned}
& r_{1}(k) \\
& =0.94 y(k-1)+3.17 \cdot 10^{-2} y(k-2) \\
& \quad+1.8 \cdot 10^{-2} y(k-3)-10.1 u_{1}(k-1) \\
& \quad+4.08 u_{1}(k-2) \\
& \quad+6 u_{1}(k-3)+8.74 \cdot 10^{-2} u_{2}(k-1) \\
& \quad-0.26 u_{2}(k-2)+0.18 u_{2}(k-3) \\
& \quad-3.23 u_{3}(k-1)+2.37 u_{3}(k-2) \\
& \quad+0.85 u_{3}(k-3)-3.84 \cdot 10^{-2} u_{5}(k-1) \\
& \quad+9.10 \cdot 10^{-2} u_{5}(k-2)+1.72 \cdot 10^{-2} u_{5}(k-3) \\
& \quad-6.99-y(k),
\end{aligned}
$$


Rule 4:

$$
\begin{aligned}
& r_{1}(k) \\
& =0.97 y(k-1)+3.01 \cdot 10^{-2} y(k-2) \\
& \quad+1.14 \cdot 10^{-2} y(k-3)+2.97 u_{1}(k-1) \\
& \quad-5.10 u_{1}(k-2)+1.99 u_{1}(k-3) \\
& \quad+1.38 \cdot 10^{-2} u_{2}(k-1) \\
& \quad-1.56 \cdot 10^{-2} u_{2}(k-2)-2.12 \cdot 10^{-3} u_{2}(k-3) \\
& \quad+1.75 u_{3}(k-1)+0.35 u_{3}(k-2) \\
& \quad-2.03 u_{3}(k-3)-0.27 u_{5}(k-1) \\
& \quad-0.34 u_{5}(k-2) \\
& \quad+0.62 u_{5}(k-3)-1.23-y(k),
\end{aligned}
$$

\section{Rule 5:}

$$
\begin{aligned}
& r_{1}(k) \\
& =0.92 y(k-1)+7.75 \cdot 10^{-2} y(k-2) \\
& \quad+5.99 \cdot 10^{-3} y(k-3)+6.54 u_{1}(k-1) \\
& \quad-2.84 u_{2}(k-2)-3.82 u_{1}(k-3) \\
& \quad-4.95 \cdot 10^{-2} u_{2}(k-1) \\
& \quad+6.97 \cdot 10^{-2} u_{2}(k-2)-2.66 \cdot 10^{-2} u_{2}(k-3) \\
& \quad+4.44 u_{3}(k-1)-0.89 u_{3}(k-2) \\
& \quad-3.28 u_{3}(k-3)+0.41 u_{5}(k-1)-1.15 u_{5}(k-2) \\
& \quad+0.75 u_{5}(k-3)-1.65-y(k),
\end{aligned}
$$

\section{Rule 6:}

$$
\begin{aligned}
& r_{1}(k) \\
& =0.93 y(k-1)+4.43 \cdot 10^{-2} y(k-2) \\
& \quad+1.46 \cdot 10^{-2} y(k-3)+1.87 \cdot 10^{-2} u_{1}(k-1) \\
& \quad+9.39 \cdot 10^{-2} u_{1}(k-2)-7.07 \cdot 10^{-2} u_{1}(k-3) \\
& \quad+6.48 \cdot 10^{-2} u_{2}(k-1)-7.11 \cdot 10^{-2}, u_{2}(k-2) \\
& \quad+1.09 \cdot 10^{-2} u_{2}(k-3)+2.62 u_{3}(k-1) \\
& \quad-1.61 u_{3}(k-2)-0.92 u_{3}(k-3) \\
& \quad+0.18 u_{5}(k-1)-0.65 u_{5}(k-2) \\
& \quad+0.51 u_{5}(k-3)-12.4-y(k),
\end{aligned}
$$

\section{Rule 7:}

$$
\begin{aligned}
& r_{1}(k) \\
& =1.07 y(k-1)-9.76 \cdot 10^{-2} y(k-2) \\
& \quad+2.79 \cdot 10^{-2} y(k-3)-0.38 u_{1}(k-1) \\
& \quad+0.46 u_{1}(k-2)-0.14 u_{1}(k-3) \\
& \quad+3.27 \cdot 10^{-3} u_{2}(k-1) \\
& \quad+9.10 \cdot 10^{-2} u_{2}(k-2) \\
& \quad-9.84 \cdot 10^{-2} u_{2}(k-3) \\
& \quad-1.31 u_{3}(k-1)+1.23 u_{3}(k-2) \\
& \quad+0.25 u_{3}(k-3)-3.29 u_{5}(k-1) \\
& \quad+2.16 u_{5}(k-2)+1.15 u_{5}(k-3) \\
& \quad-2.38-y(k) .
\end{aligned}
$$

On the other hand, the structure of the fuzzy TS prototype for the generation of the residual $r_{2}(k)$, which depends on $y(k), u_{1}(k), u_{2}(k), u_{3}(k), u_{4}(k)$, and $u_{5}(k)$, is reported in the following expressions for each rule, with $K=7$ and $n=3$ :

Rule 1:

$$
\begin{aligned}
& r_{2}(k) \\
& =1.01 y(k-1)+6.55 \cdot 10^{-2} y(k-2)
\end{aligned}
$$

$$
\begin{aligned}
& -7.19 \cdot 10^{-2} y(k-3)-5.06 u_{1}(k-1) \\
& +3.01 u_{1}(k-2)+2.03 u_{1}(k-3) \\
& +0.21 u_{2}(k-1)-0.17 u_{2}(k-2) \\
& +1.67 \cdot 10^{-2} u_{2}(k-3)-2.16 u_{3}(k-1) \\
& +0.19 u_{3}(k-2)+1.95 u_{3}(k-3) \\
& +0.93 u_{4}(k-1)+1.84 u_{4}(k-2) \\
& -2.86 u_{4}(k-3)+5.29 \cdot 10^{-2} u_{5}(k-1) \\
& 0.39 u_{5}(k-2)-0.5 u_{5}(k-3)-139-y(k),
\end{aligned}
$$

Rule 2:

$r_{2}(k)$

$$
\begin{aligned}
= & 1.07 y(k-1)-1.79 \cdot 10^{-2} y(k-2) \\
& -5.11 \cdot 10^{-2} y(k-3)-0.14 u_{1}(k-1) \\
& -0.74 u_{1}(k-2)+0.89 u_{1}(k-3) \\
& +4.07 \cdot 10^{-3} u_{2}(k-1) \\
& +3.68 \cdot 10^{-3} u_{2}(k-2)-1.07 \cdot 10^{-2} u_{2}(k-3) \\
& +0.49 u_{3}(k-1)+0.74 u_{3}(k-2) \\
& -1.22 u_{3}(k-3)+0.94 u_{4}(k-1) \\
& -1.13 u_{4}(k-2)+9.46 \cdot 10^{-2} u_{4}(k-3) \\
& -0.96 \cdot u_{5}(k-1)+1.02 u_{5}(k-2) \\
& -7.86 \cdot 10^{-2} u_{5}(k-3)+11.2-y(k),
\end{aligned}
$$

Rule 3:

$$
\begin{aligned}
& r_{2}(k) \\
& =0.87 y(k-1)+4.42 \cdot 10^{-2} y(k-2) \\
& \quad+8.66 \cdot 10^{-2} y(k-3)+0.84 u_{1}(k-1) \\
& \quad+7.18 \cdot 10^{-1} u_{1}(k-2)-1.81 u_{1}(k-3) \\
& \quad-8.45 \cdot 10^{-2} u_{2}(k-1)-1.16 \cdot 10^{-2} u_{2}(k-2) \\
& \quad+9.07 \cdot 10^{-2} u_{2}(k-3)+1.35 u_{3}(k-1) \\
& \quad-0.59 u_{3}(k-2)-0.45 u_{3}(k-3)-1.88 u_{4}(k-1) \\
& \quad+1.29 u_{4}(k-2)+0.7 u_{4}(k-3)+0.68 u_{5}(k-1) \\
& \quad-1.47 u_{5}(k-2)+0.88 u_{5}(k-3)-9.04-y(k),
\end{aligned}
$$

Rule 4:

$$
\begin{aligned}
& r_{2}(k) \\
& =0.95 y(k-1)-5.55 \cdot 10^{-2} y(k-2) \\
& \quad+7.72 \cdot 10^{-2} y(k-3) \\
& \quad+1.67 u_{1}(k-1)-0.71 u_{1}(k-2) \\
& \quad-0.89 u_{1}(k-3)+3.24 \cdot 10^{-2} u_{2}(k-1) \\
& \quad-3.62 \cdot 10^{-2} u_{2}(k-2)-7.09 \cdot 10^{-4} u_{2}(k-3) \\
& \quad-2.16 u_{3}(k-1)-2.40 u_{3}(k-2)+4.35 u_{3}(k-3) \\
& \quad+0.46 u_{4}(k-1)-1.42 u_{4}(k-2)+1.12 u_{4}(k-3) \\
& \quad+2.14 u_{5}(k-1)-0.88 u_{5}(k-2)-1.18 u_{5}(k-3) \\
& \quad+25.5-y(k),
\end{aligned}
$$

\section{Rule 5:}

$$
\begin{aligned}
& r_{2}(k) \\
& =0.8 y(k-1)+0.16 y(k-2) \\
& \quad+8.05 \cdot 10^{-2} y(k-3)+6.32 u_{1}(k-1) \\
& \quad-31 u_{1}(k-2)+24.4 u_{1}(k-3) \\
& \quad+1.72 \cdot 10^{-2} u_{2}(k-1)+0.14 u_{2}(k-2) \\
& \quad-0.14 u_{2}(k-3)+12.2 u_{3}(k-1) \\
& \quad+3.87 u_{3}(k-2)-15.3 u_{3}(k-3) \\
& \quad-1.22 u_{4}(k-1)+2.74 u_{4}(k-2)-2.26 u_{4}(k-3) \\
& \quad-2.87 u_{5}(k-1)-0.4 u_{5}(k-2)+3.23 u_{5}(k-3) \\
& \quad-46.4-y(k),
\end{aligned}
$$




$$
\begin{aligned}
& \text { Rule 6: } \\
& \begin{aligned}
r_{2}(k) \\
=y(k-1)-1.80 \cdot 10^{-2} y(k-2) \\
\quad+1.51 \cdot 10^{-2} y(k-3)+0.18 u_{1}(k-1) \\
\quad+5.57 \cdot 10^{-2} u_{1}(k-2)-0.25 u_{1}(k-3) \\
\quad+8.68 \cdot 10^{-3} u_{2}(k-1)+4.53 \cdot 10^{-3} u_{2}(k-2) \\
\quad+4.99 \cdot 10^{-3} u_{2}(k-3)+6.74 \cdot 10^{-2} u_{3}(k-1) \\
\quad-2.77 \cdot 10^{-2} u_{3}(k-2)-2.78 \cdot 10^{-2} u_{3}(k-3) \\
\quad-2.55 \cdot 10^{-3} u_{4}(k-1)-7.63 \cdot 10^{-2} u_{4}(k-2) \\
\quad-2.67 \cdot 10^{-2} u_{4}(k-3)-0.51 u_{5}(k-1) \\
\quad+0.19 u_{5}(k-2)+0.31 u_{5}(k-3)+6.53-y(k),
\end{aligned}
\end{aligned}
$$

\section{Rule 7:}

$$
\begin{aligned}
& r_{2}(k) \\
& =0.93 y(k-1)-5.66 \cdot 10^{-2} y(k-2) \\
& \quad+0.14 y(k-3)+2.87 u_{1}(k-1)+0.33 u_{1}(k-2) \\
& \quad-3.5 u_{1}(k-3)-0.19 u_{2}(k-1)+0.27 u_{2}(k-2) \\
& \quad-0.13 u_{2}(k-3)+3.28 u_{3}(k-1)-0.45 u_{3}(k-2) \\
& \quad-2.14 u_{3}(k-3)-1.48 u_{4}(k-1)-2.17 u_{4}(k-2) \\
& \quad+4.81 u_{4}(k-3)-4.37 u_{5}(k-1)-0.13 u_{5}(k-2) \\
& \quad+4.64 u_{5}(k-3)-22.9-y(k) .
\end{aligned}
$$

Finally, the structure of the fuzzy TS prototype for the generation of the residual $r_{3}(k)$, which uses the signals $y(k), u_{1}(k), u_{2}(k), u_{3}(k), u_{5}(k)$, and $u_{6}(k)$, is reported in the expressions for each rule, with $K=7$ and $n=3$ :

Rule 1:

$$
\begin{aligned}
& r_{3}(k) \\
& =0.94 y(k-1) \\
& \quad+6.36 \cdot 10^{-2} y(k-2)+6.19 \cdot 10^{-3} y(k-3) \\
& \quad+6.18 u_{1}(k-1)-5.44 u_{1}(k-2) \\
& \quad-0.89 u_{1}(k-3)+3.32 \cdot 10^{-2} u_{2}(k-1) \\
& \quad-8.79 \cdot 10^{-2} u_{2}(k-2)+5.08 \cdot 10^{-2} u_{2}(k-3) \\
& \quad+2.67 u_{3}(k-1)-0.24 u_{3}(k-2) \\
& \quad-2.28 u_{3}(k-3)+0.48 u_{5}(k-1) \\
& \quad-1.26 u_{5}(k-2)+0.79 u_{5}(k-3) \\
& \quad-0.35 u_{6}(k-1)+1.27 u_{6}(k-2) \\
& \quad-0.93 u_{6}(k-3)-1.98-y(k),
\end{aligned}
$$

Rule 2:

$$
\begin{aligned}
& r_{3}(k) \\
& =0.88 y(k-1)+0.16 y(k-2) \\
& \quad-4.36 \cdot 10^{-2} y(k-3)+1.13 u_{1}(k-1) \\
& \quad-2.21 u_{1}(k-2)+1.11 u_{1}(k-3) \\
& \quad-1.81 \cdot 10^{-2} u_{2}(k-1)-0.28 u_{2}(k-2) \\
& \quad+0.32 u_{2}(k-3)+1.91 u_{3}(k-1) \\
& \quad-2.34 u_{3}(k-2)+0.63 u_{3}(k-3) \\
& \quad+1.87 u_{5}(k-1)-1.51 u_{5}(k-2) \\
& \quad-0.34 u_{5}(k-3)+0.35 u_{6}(k-1) \\
& \quad-0.48 u_{6}(k-2)+0.13 u_{6}(k-3) \\
& \quad-70.9-y(k),
\end{aligned}
$$

Rule 3:

$r_{3}(k)$

$=0.96 y(k-1)$

$$
\begin{aligned}
& -2.40 \cdot 10^{-2} y(k-2)+6.28 \cdot 10^{-2} y(k-3) \\
& +0.77 u_{1}(k-1)-8.32 u_{1}(k-2) \\
& +7.5 u_{1}(k-3)-8.94 \cdot 10^{-2} u_{2}(k-1) \\
& +0.18 u_{2}(k-2)-9.69 \cdot 10^{-2} u_{2}(k-3) \\
& +7.22 u_{3}(k-1)-2.35 u_{3}(k-2) \\
& -4.51 u_{3}(k-3)-1.17 u_{5}(k-1) \\
& +0.35 u_{5}(k-2)+0.85 u_{5}(k-3) \\
& +0.46 u_{6}(k-1)+2.17 u_{6}(k-2) \\
& -2.56 u_{6}(k-3)-5.17-y(k),
\end{aligned}
$$

Rule 4:

$$
\begin{aligned}
& r_{3}(k) \\
& =y(k-1)-4.86 \cdot 10^{-2} y(k-2) \\
& \quad 4.44 \cdot 10^{-2} y(k-3)-1.7 u_{1}(k-1) \\
& \quad+3.31 u_{1}(k-2)-1.51 u_{1}(k-3) \\
& \quad-9.95 \cdot 10^{-3} u_{2}(k-1)+0.25 u_{2}(k-2) \\
& \quad-0.25 u_{2}(k-3)-0.95 u_{3}(k-1) \\
& \quad+1.87 u_{3}(k-2)-0.97 u_{3}(k-3) \\
& \quad-0.67 u_{5}(k-1)+0.25 u_{5}(k-2) \\
& \quad+0.38 u_{5}(k-3)-0.53 u_{6}(k-1) \\
& \quad+1.02 u_{6}(k-2)-0.48 u_{6}(k-3)+11.1-y(k),
\end{aligned}
$$

Rule 5:

$$
\begin{aligned}
& r_{3}(k) \\
& =0.99 y(k-1) \\
& \quad+2.93 \cdot 10^{-3} y(k-2)+6.6 \cdot 10^{-3} y(k-3) \\
& \quad+3.06 u_{1}(k-1)-3.11 u_{1}(k-2) \\
& \quad+7.23 \cdot 10^{-2} u_{1}(k-3)+9.51 \cdot 10^{-3} u_{2}(k-1) \\
& \quad-9.02 \cdot 10^{-3} u_{2}(k-2)-2.82 \cdot 10^{-3} u_{2}(k-3) \\
& \quad-0.71 u_{3}(k-1)+0.4 u_{3}(k-2) \\
& \quad+0.41 u_{3}(k-3)-0.98 u_{5}(k-1) \\
& \quad+0.26 u_{5}(k-2)+0.76 u_{5}(k-3) \\
& \quad+0.23 u_{6}(k-1)-0.21 u_{6}(k-2) \\
& \quad-5.73 \cdot 10^{-3} u_{6}(k-3)-2.06-y(k),
\end{aligned}
$$

Rule 6:

$$
\begin{aligned}
& r_{3}(k) \\
& =0.97 y(k-1) \\
& \quad-2.10 \cdot 10^{-2} y(k-2)+3.56 \cdot 10^{-2} y(k-3) \\
& \quad-25.1 u_{1}(k-1)+24 u_{1}(k-2) \\
& \quad+1.22 u_{1}(k-3)+7.21 \cdot 10^{-2} u_{2}(k-1) \\
& \quad+6.72 \cdot 10^{-2} u_{2}(k-2)-0.14 u_{2}(k-3) \\
& \quad-7.18 u_{3}(k-1)+9.71 u_{3}(k-2) \\
& \quad-2.49 u_{3}(k-3)-2.25 u_{5}(k-1) \\
& \quad+2.36 u_{5}(k-2)-5.16 \cdot 10^{-2} u_{5}(k-3) \\
& \quad+0.11 u_{6}(k-1)-1.86 u_{6}(k-2) \\
& \quad+1.37 u_{6}(k-3)+36.6-y(k),
\end{aligned}
$$

Rule 7:

$r_{3}(k)$

$$
\begin{aligned}
& =1.18 y(k-1) \\
& \quad-0.19 y(k-2)+2.16 \cdot 10^{-2} y(k-3) \\
& \quad+0.41 u_{1}(k-1)-0.75 u_{1}(k-2) \\
& \quad+0.27 u_{1}(k-3)+2.9 \cdot 10^{-2} u_{2}(k-1) \\
& \quad+5.69 \cdot 10^{-4} u_{2}(k-2)-3.61 \cdot 10^{-2} u_{2}(k-3) \\
& \quad-0.27 u_{3}(k-1)-0.23 u_{3}(k-2)
\end{aligned}
$$




$$
\begin{aligned}
& +0.85 u_{3}(k-3)-4.5 u_{5}(k-1)+4.43 u_{5}(k-2) \\
& +0.13 u_{5}(k-3)+0.32 u_{6}(k-1) \\
& -0.85 u_{6}(k-2)+0.53 u_{6}(k-3)-13-y(k) .
\end{aligned}
$$

The FDI scheme capabilities were then validated by testing it on various real data sets, acquired from a Jeep Wrangler under an emission test, according the European Union Driving Cycle (EUDC). It is assumed, in general, that faulty data can be "logically" separable from fault-free sequences generated by the process under diagnosis.

By considering different test data sequences, Table 6 reports the Predicted Per Cent Reconstruction Error (PPCRE), where the reconstruction error $r_{i}(k)$ in fault-free conditions is computed as the difference between the actual diesel engine output $y(k)$ and the output from the $i$-th residual generator. Since this error is normalised with respect to the output standard deviation, it can be seen as the percentage of data that are not correctly explained by the identified TS models. The results summarised in Table 6 indicate that the fuzzy prototypes are able to generate reliable residual signals for real diesel engine fault diagnosis.

Table 6. TS fuzzy model errors for different data sets.

\begin{tabular}{|l|ccc|}
\hline Data set & \multicolumn{3}{|c|}{ PPCRE } \\
& $r_{1}(k)$ & $r_{2}(k)$ & $r_{3}(k)$ \\
\hline \hline estimation data & $0.90 \%$ & $0.87 \%$ & $0.92 \%$ \\
validation data & $2.80 \%$ & $1.80 \%$ & $2.10 \%$ \\
test data & $4.20 \%$ & $3.50 \%$ & $4.00 \%$ \\
\hline
\end{tabular}

Using these identified TS fuzzy prototypes, the diesel engine FDI scheme design has been applied to the actual process, as shown in Section 6.2, whilst further experiments are summarised in Section 6.3

6.2. Real-time diagnostic implementation. Clearly, the presented fuzzy identification would require a considerable calculation effort if it were implemented on-line. State-of-the-art Engine Control Units (ECUs) would not be able to perform these algorithms in an appropriate time. Assuming that the growth in calculation power has been proceeding at high speed of the last few years, future ECUs should make sufficient calculation time available within some years, where very simple adaptation or identification algorithms could be implemented. However, it is worth noticing that the complete fuzzy modelling oriented to the design of the FDI strategy suggested in this work was computed off-line.

On the other hand, special real-time computer systems based on digital signal processors already allow implementation and testing of the model-based designs in vehicles or an engine test stand. In order to operate the designed fuzzy predictors for an FDI purpose under realistic fault and working conditions, a real-time system was implemented at a dynamic engine test stand where it could be run parallel to the production car's ECU. This system uses the production car sensors, and the input-output messages of the ECU.

The actuated signals, which are calculated in real-time, are then sent to the actual actuators by means of a suitable electronic interface. On the other hand, output measurements can be easily acquired from the ECU system. Thus, the complete system, whose logic diagram is reported in Fig. 20, allows testing the capabilities of the suggested fuzzy FDI scheme, when working in connection with a real process.

In this application, the test system is used as a rapid prototyping environment. The goal of this structure is to enable very fast and easy implementation and testing of the new FDI concepts on real-time hardware. The user is enabled to code newly developed FDI algorithms from block diagrams via the Matlab ${ }^{\circledR}$ and Simulink ${ }^{\circledR}$ environments, and download the code by means of an automatic code generation software to real-time hardware (e.g., the well-known Real-Time Workshop of Matlab ${ }^{\circledR}$ ). In particular, the developed TS fuzzy prototypes were implemented as simple look-up tables, which can be easily stored on the car's ECU.

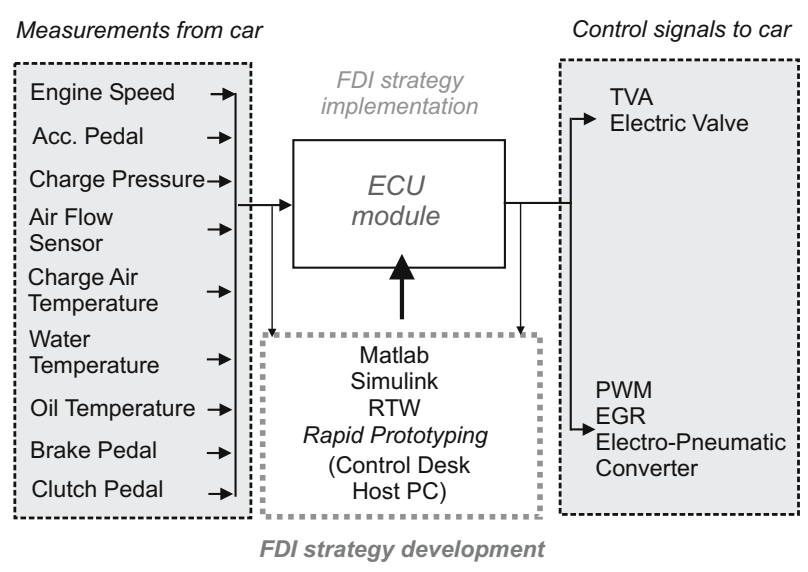

Fig. 20. Real-time validation of the FDI strategy with the test vehicle.

Therefore, the described real-time hardware system represented in Fig. 20 has enabled viable implementation, validation, and testing of the proposed FDI strategy, whose results have been shown in Section 6.3 Further experiments used for assessing the effectiveness of the FDI approach are shown in Sections 6.4 and 6.5

6.3. FDI results. The following experimental results have been obtained by considering the faults described in 
Section 3.1 and implemented in real-time, as described in Section 6.2 They cause alteration of the signals $u_{i}(k)$ and $y(k)$, and therefore of the residuals $r_{i}(k)$ given by the predictive models in the form of Eqn. (8). Residuals indicate fault occurrence according to the logic of Eqn. 17), whether their values are lower or higher than the thresholds fixed in fault-free conditions.

As an example, Figs. 21, 23 and 25 show the results from the application of the fuzzy prototypes for residual generation.

In particular, Fig. 21 shows the fault-free and the faulty residuals $r_{1}(k)$ concerning the fault case 1 , which commences at $t=850 \mathrm{~s}$, with a rate of $1 \%$ per hour. The fault detection thresholds highlighted in Fig. 21 with dotted lines, have been settled according to Eqns. (17) with a $\delta=4.8$. This choice allows achieving fault detection of $750 \mathrm{~s}$.

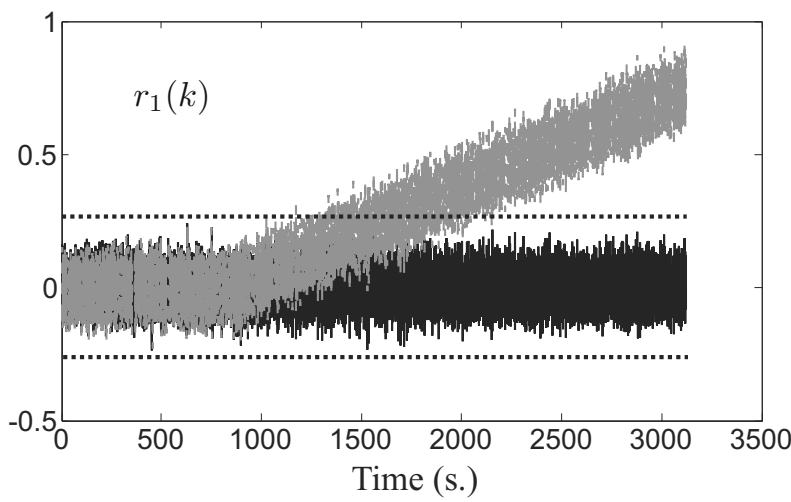

Fig. 21. Fault-free (continuous black) and faulty (dashed grey) residuals for the fault case 1 .

In the same situation, Fig. 22 depicts the residual signals $r_{2}(k)$ and $r_{3}(k)$ that are generated by the fuzzy generators insensitive to the fault case 1 .

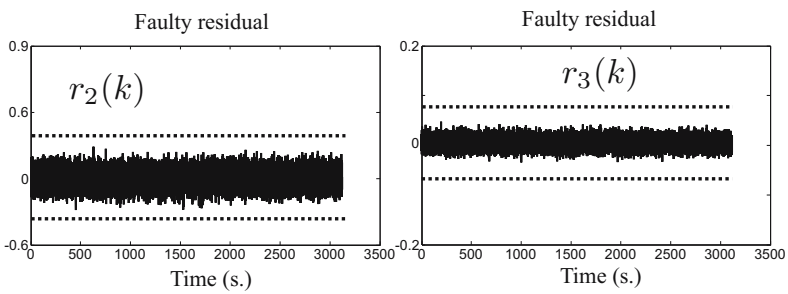

Fig. 22. Residuals $r_{2}(k)$ and $r_{3}(k)$ from the generators insensitive to the fault case 1 .

On the other hand, Fig. 23 shows the fault-free and faulty residual signals regarding the temperature sensor of the diesel engine, i.e., the fault case 2. This temperature sensor fault described in Section 3.1 is linked to the signal $f_{u}(t)$, which models the fault affecting the engine variable $u_{4}$. The fault commences at $t=840 \mathrm{~s}$., and influences the $u_{4}$ signal, with a rate of $5 \%$ per hour.

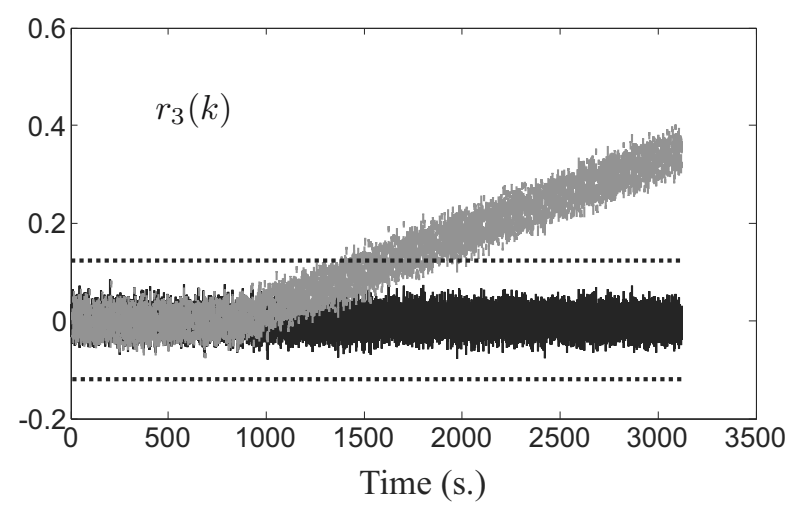

Fig. 23. Fault-free (black continuous) and faulty (grey dashed) residuals for the fault case 2 .

In the same condition, Fig. 24 depicts the residual signals $r_{1}(k)$ and $r_{2}(k)$ that are generated by the fuzzy generators insensitive to the fault case 2 .
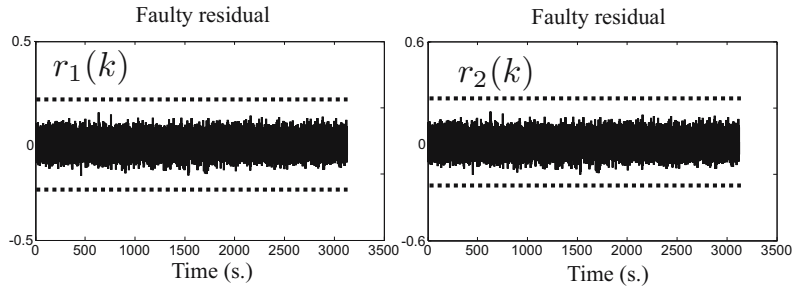

Fig. 24. Residuals $r_{1}(k)$ and $r_{2}(k)$ from the generators insensitive to the fault case 2 .

The non-zero values of the residuals in fault-free conditions shown in Figs. 21 and 23 are due to modelling and measurement errors. A value of $\delta=4.5$ has been selected, according to the procedure described in Sections 5 and 6.5 , thus guaranteeing a fault detection delay of about $950 \mathrm{~s}$. Figure 23 reports also fault detection thresholds depicted using dotted lines.

Finally, Fig. 25 depicts the residuals corresponding to the fault case 3 , which commences at $t=850 \mathrm{~s}$. The residuals are computed by means of the fuzzy predictor monitoring the TVA control signal $u_{6}(k)$. A choice of $\delta=4.6$ allows detecting a fault with a rate of $7 \%$ per hour after $1000 \mathrm{~s}$.

In the same situation, Fig. 26 depicts the residual signals $r_{2}(k)$ and $r_{3}(k)$ generated by the fuzzy generators of the bank that are insensitive to the fault case 3 .

Note that the fault detection thresholds reported in the relation of Eqn. (17) are represented as constant dotted lines in Figs. 21 26. Their values were properly settled by selecting a proper $\delta$, which leads to minimising the false alarm and missed fault rates while maximising the 


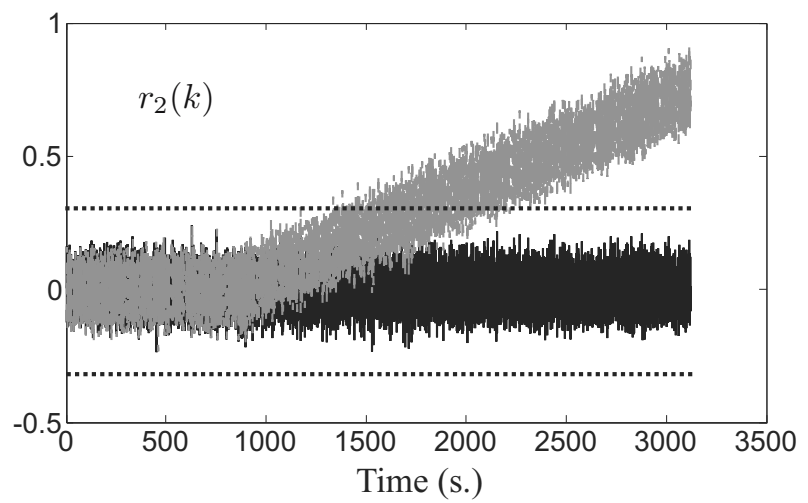

Fig. 25. Fault-free (continuous black) and faulty (dashed grey) residuals for the fault case 3 .

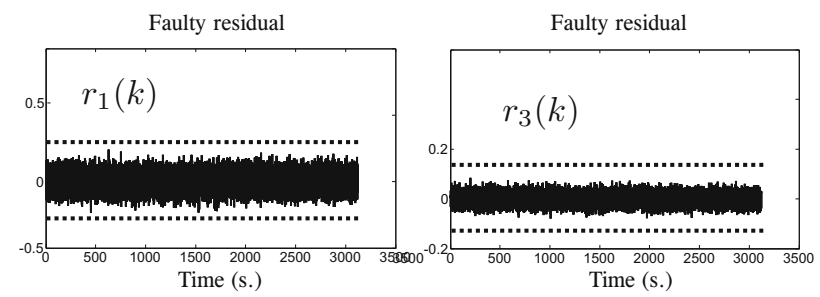

Fig. 26. Residuals $r_{1}(k)$ and $r_{3}(k)$ from the generators insensitive to the fault case 3 .

correct detection and isolation rates. The optimisation procedure for the selection of the parameter $\delta$ is further discussed in Section 6.5. These parameters depend on the automotive application considered. In these conditions, the fault is correctly detected when the corresponding residual signals exceed the thresholds by a fixed number of consecutive samples.

Finally, it is worth observing that the developed strategy based on fuzzy prototypes allows detecting and isolating realistic faults using uncertain measurements acquired from the real diesel engine test-rig. Moreover, the real-time hardware system described in Section 6.2 enabled the implementation, validation, and assessment of the proposed FDI strategy, thus proving its reliability and robustness when applied also to real data. However, further investigations are shown in Sections 6.4 and 6.5.

6.4. Comparative studies. This section provides some comparative results with respect to another FDI scheme, in particular, the one relying on a bank of Unknown Input Kalman Filters (UIKFs) (Chen and Patton, 1999). The UIKF bank was designed on the basis of a linear state-space 15 -th order model of the diesel engine, derived on the basis of the identification method presented, e.g., by Simani et al. (2000) and Simani (2007), but without exploiting the fuzzy multiple-model identification.
It is worth noticing that the state-space linear model of 15-th order was computed using a black-box identification procedure exploiting the subspace N4SID algorithm (Ljung, 1999). It is clear that, with reference to this identified black-box state-space model, the state variables do not have any physical meaning. In fact, the model order was selected for achieving good estimation properties. Moreover, this model was not derived from any linearisation procedure, which motivates again the lack of any physical meaning of the state vector. The model structure was validated using the tools available from the System Identification Toolbox (Ljung, 1997) developed in the Matlab ${ }^{\circledR}$ environment. The validation is based on the auto- and cross-correlation analysis of the model estimation error, as described again by Ljung (1999). The suggested comparison between the UIKF approach and fuzzy residual generators seems appropriate indeed. In fact, even if UIKFs are designed on the basis of the linear state-space model, they allow decoupling both the model-reality mismatch and the measurement errors. In this way, UIKFs organised into a bank scheme for residual generation are compared with TS fuzzy models.

Once the linear state-space 15-th order model for the diesel engine has been computed, the UIKFs of the bank have been obtained by the design technique described, e.g., by Chen and Patton (1999, Section 3.5.1, pp. 99-108), while the noise covariance matrices have been estimated as described by Simani et al. (2001, Chapter 4, pp. 117-131). In particular, the design of the UIKFs of the bank is enhanced by the estimation of the noise covariance constant matrices directly achieved from the Frisch scheme, already exploited here, and recalled in Section 4.1.2 Using the notation of Chen and Patton (1999, Section 3.5.1, p. 100), with the knowledge of the (constant) noise covariance matrices $Q_{k}$ and $R_{k}$, which are computed from the identified $\left(\overline{\tilde{\sigma}}_{u}, \overline{\tilde{\sigma}}_{y}\right)$ (Simani, 2007), the time-varying gain $K_{k}$ and error covariance $P_{k}$ matrices of the Kalman filter bank are computed. Therefore, the motivation for using the bank of non-stationary Kalman filter with unknown inputs here is twofold. First, the time-varying (non-stationary) Kalman filters designed on the basis of the linear state-space model of the diesel engine are able to generate suitable residuals for the nonlinear system. In practice, in this situation the identified variance noise values $\left(\overline{\tilde{\sigma}}_{u}, \overline{\tilde{\sigma}}_{y}\right)$ take into account the uncertainty due to both modelling and measurement errors, i.e., the disturbance term of the UIKFs (Simani et al., 1999; Fantuzzi et al., 2002). Secondly, the bank of UIKFs allows us to achieve also the required fault isolation task, in the same way exploited for the design of the bank of fuzzy estimators, as described in Section 5 .

As an example, the FDI residuals generated via the UIKF bank are shown in Fig. 27 In particular, the UIKF bank residuals relative to $2150 \mathrm{~s}$. simulation time are depicted in Fig. 27, when the fault cases 1, 2, and 3 
commence at $850 \mathrm{~s}$.

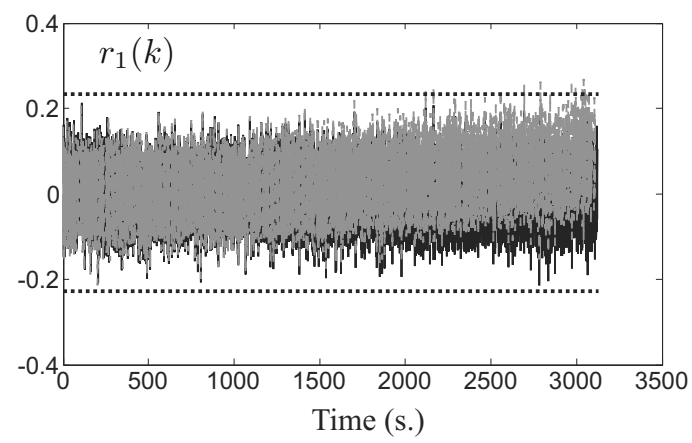

(a)

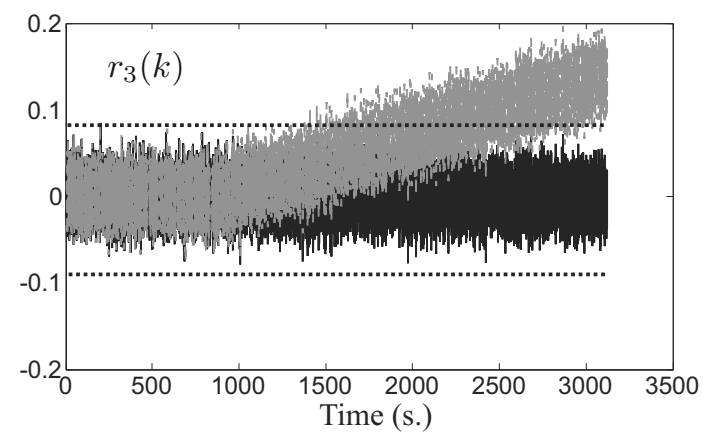

(b)

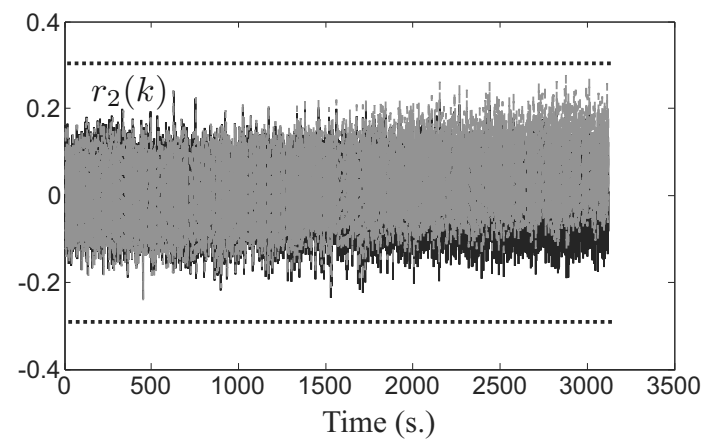

(c)

Fig. 27. UIKF fault-free (continuous black) and faulty (dashed grey) residuals: case 1 fault residuals (a), case 2 fault residuals (b), case 3 fault residuals (c).

The residual signals of Fig. 27 are different from those shown in the previous section. In this case in fact, the residual signals generated via the UIKF bank do not allow achieving the detection of the fault cases 1 and 3 . Moreover, the detection of the fault case 2 is achievable only if its rate is increased. Table7 7 summarises the results obtained by comparing the FDI technique recalled in this subsection.

A few comments can be drawn here. When the modelling of the dynamic system can be perfectly obtained, model-based schemes are preferred. In some situations, the UIKF can take advantage of its noise and disturbance rejection capabilities, but with quite
Table 7. Minimal detectable faults.

\begin{tabular}{|c|c|c|}
\hline Fault case & UIKF & Fuzzy approach \\
\hline \hline Case 1 & Not detectable & $1 \%$ \\
\hline Case 2 & $15 \%$ & $5 \%$ \\
\hline Case 3 & Not detectable & $7 \%$ \\
\hline
\end{tabular}

complicated and not straightforward design procedures. However, in this case, the fault sensitivity of the linear UIKF is lower with respect to nonlinear fuzzy predictors. On the other hand, regarding the developed FDI fuzzy method, it seems rather simple when developed in connection with fuzzy identification, even if optimisation stages are required, for example, for optimal FDI threshold selection. This further issue has been described in Section 6.5

6.5. FDI scheme robustness evaluation. In this section, further experimental results have been reported regarding the performance optimisation and evaluation of the developed FDI scheme with respect to modelling errors and measurement uncertainty. In particular, the simulation of different fault-free and faulty data sequences has been performed by exploiting the test-rig setup of Section 6.2 and a Matlab ${ }^{\circledR}$ Monte-Carlo analysis. In fact, the Monte-Carlo tool is useful at this stage as FDI performances depend the residual error magnitude as well as on the measured signal $u(k)$ and $y(k)$ errors. As remarked in Section 6.2, the experimental test-rig is able to generate the required real-time signals and the injection of realistic fault cases.

Moreover, as described in Section 5, it is assumed that the input-output data $u(k)$ and $y(k)$ were affected by measurement errors. Thus, for performance evaluation and reliability analysis of the FDI scheme, some indices have been used. The performances of the FDI method are thus empirically evaluated on 500 Monte-Carlo runs, corresponding to the same number of driving cycle tests. These indices are defined as follows:

False Alarm Rate $\left(r_{f a}\right)$ : the number of wrongly detected faults divided by total fault cases;

Missed Fault Rate $\left(r_{m f}\right)$ : for each fault, the total number of undetected faults, divided by the total number of times that the fault case occurs;

True Detection/Isolation Rate $\left(r_{t d}, r_{t i}\right)$ : for a particular fault case, the number of times it is correctly detected/isolated, divided by total number of times that the fault case occurs;

Mean Detection/Isolation Delay $\left(\tau_{m d}, \tau_{m i}\right)$ : for a particular fault case, the average detection/isolation delay time. 
These criteria are computed off-line for each fault case. Table 8 summarises the results obtained by considering the fuzzy residual generators, and with a choice of the threshold parameter $\delta$ in Eqns. (17) leading to achieve optimal results.

Table 8. Monte-Carlo analysis by monitoring residuals via Eqns. (17) with optimal $\delta$.

\begin{tabular}{|c||ccccc|}
\hline Fault & $r_{f a}$ & $r_{m f}$ & $r_{t d}, r_{t i}$ & $\tau_{m d}, \tau_{m i}$ & $\delta$ \\
\hline \hline Case 1 & 0.002 & 0.003 & 0.997 & $750 \mathrm{~s}$ & 4.8 \\
\hline Case 2 & 0.001 & 0.001 & 0.999 & $950 \mathrm{~s}$ & 4.5 \\
\hline Case 3 & 0.002 & 0.003 & 0.997 & $1000 \mathrm{~s}$ & 4.6 \\
\hline
\end{tabular}

Table 8 shows that with the proper selection of the threshold levels depending on $\delta$ it is possible to achieve false alarm and missed fault rates of less than $0.3 \%$ and detection and isolation rates larger than $99.7 \%$, with minimal detection and isolation delay times. The results demonstrate also that Monte-Carlo analysis is an effective tool for experimentally testing the design robustness of the proposed FDI method with respect to error and uncertainty. This last simulation technique example hence facilitates an assessment of the reliability of the developed FDI method applied to real test cases.

\section{Conclusion}

The paper provided practical results in the diagnosis of faults on a real diesel engine process by using a model-based approach. The reported results show that faults having barely detectable effects on measurements, can be detected using residual generators in the form of dynamic fuzzy Takagi-Sugeno prototypes. The outlined method focused to some extent on identified input-output models, thus the final fault diagnosis algorithm is based only on input-output processing of all measurable signals.

The paper suggested to exploit identified piecewise-affine models, although the system considered is strongly nonlinear. This is regarded as important to avoid the complexities otherwise inevitable when purely nonlinear models are used. The algorithmic simplicity can be seen as a very important aspect when considering the need for verification and validation of a demonstrable scheme for industrial certification, mainly important in automotive fields. This aspect of the work, together with the fact that the modelling uncertainty and the measurement noise seem to be well tackled, confirms the straightforward application of the proposed fault diagnosis schemes to real automotive applications. Finally, the robustness and reliability properties were investigated via extensive Monte-Carlo experiments.

\section{Acknowledgment}

The author would like to express his gratitude to VM Motors S.p.A. (Cento, Ferrara, Italy) for the financial support, fruitful discussions, cooperation, and facilitation of the present research study.

A preliminary version of this paper was presented at the DPS'2011 Conference (Zamość, Poland, 2011) as a plenary talk.

\section{References}

Babuška, R. (1998). Fuzzy Modeling for Control, Kluwer Academic Publishers, Boston, MA.

Babuška, R. (2000). Fuzzy Modelling and Identification Toolbox, Version 3.1, Control Engineering Laboratory, Faculty of Information Technology and Systems, Delft University of Technology, Delft, http: / / lcewww.et.tudelft.nl/ babuska

Bonfè, M., Castaldi, P., Mimmo, N. and Simani, S. (2011). Active fault tolerant control of nonlinear systems: The cart-pole example, International Journal of Applied Mathematics and Computer Science 21(3): 441-455, DOI: 10.2478/v10006-011-0033-y.

Bosch, R. (2006). Diesel-Engine Management, 4 Edn., Wiley, Weinheim.

Chen, J. and Patton, R.J. (1999). Robust Model-Based Fault Diagnosis for Dynamic Systems, Kluwer Academic Publishers, Boston, MA.

Ding, S.X. (2008). Model-based Fault Diagnosis Techniques: Design Schemes, Algorithms, and Tools, 1st Edn., Springer, Berlin/Heidelberg.

Fantuzzi, C. and Rovatti, R. (1996). On the approximation capabilities of the homogeneous Takagi-Sugeno model, Proceedings of the 5th IEEE International Conference on Fuzzy Systems, New Orleans, LA, USA, pp. 1067-1072.

Fantuzzi, C., Simani, S., Beghelli, S. and Rovatti, R. (2002). Identification of piecewise affine models in noisy environment, International Journal of Control 75(18): 1472-1485.

Isermann, R. (2005). Fault-Diagnosis Systems: An Introduction from Fault Detection to Fault Tolerance, 1st Edn. Springer-Verlag, Weinheim.

Jain, T., Yamè, J.J. and Sauter, D. (2012). Model-free reconfiguration mechanism for fault tolerance, International Journal of Applied Mathematics and Computer Science 22(1): 125-137, DOI: 10.2478/v10006-012-0009-6.

Korbicz, J., Koscielny, J. M., Kowalczuk, Z. and Cholewa, W. (Eds.) (2004). Fault Diagnosis: Models, Artificial Intelligence, Applications, Springer-Verlag, London.

Ljung, L. (1997). System Identification Toolbox for Use with MATLAB ${ }^{\circledR}$ User's Guide, The MathWorks, Inc., Natick, MA.

Ljung, L. (1999). System Identification: Theory for the User, 2nd Edn., Prentice Hall, Englewood Cliffs, NJ. 
Marusak, P.M. and Tatjewski, P. (2008). Actuator fault tolerance in control systems with predictive constrained set-point optimizers, International Journal of Applied Mathematics and Computer Science 18(4): 539-551, DOI: 10.2478/v10006-008-0047-2.

Pulkrabek, W.W. (2003). Engineering Fundamentals of the Internal Combustion Engine, 2nd Edn., Prentice Hall, Richmond, TX.

Rovatti, R. (1996). Takagi-Sugeno models as approximators in Sobolev norms: The SISO case, 5th IEEE International Conference on Fuzzy Systems, New Orleans LA, USA, Vol. 2, pp. 1060-1066.

Rovatti, R., Fantuzzi, C. and Simani, S. (2000). High-speed DSP-based implementation of piecewise-affine and piecewise-quadratic fuzzy systems, Signal Processing Journal 80(6): 951-963.

Simani, S. (2007). Fault diagnosis of a simulated industrial gas turbine via identification approach, International Journal of Adaptive Control and Signal Processing 21(4): 326-353, DOI: 10.1002/acs.924.

Simani, S., Fantuzzi, C. and Beghelli, S. (2000). Diagnosis techniques for sensor faults of industrial processes, IEEE Transactions on Control Systems Technology 8(5): 848-855.

Simani, S., Fantuzzi, C. and Patton, R.J. (2003). Modelbased Fault Diagnosis in Dynamic Systems Using Identification Techniques, Advances in Industrial Control, Vol. 1, Springer-Verlag, London.

Simani, S., Fantuzzi, C., Rovatti, R. and Beghelli, S. (1999). Parameter identification for piecewise linear fuzzy models in noisy environment, International Journal of Approximate Reasoning 1(22): 149-167.

Stamatis, D.H. (2003). Failure Mode and Effect Analysis: FMEA from Theory to Execution, 2nd Edn., ASQ Quality Press, Milwaukee, WI.

Svard, C. and Nyberg, M. (2010a). Residual generators for fault diagnosis using computation sequences with mixed causality applied to automotive systems, IEEE Transactions on Systems, Man and Cybernetics, Part A: Systems and Humans 40(6): 1310-1328, DOI: 10.1109/TSMCA.2010.2049993.
Svard, C. and Nyberg, M. (2010b). Residual generators for fault diagnosis using computation sequences with mixed causality applied to automotive systems, IEEE Transactions on Systems, Man and Cybernetics, Part A: Systems and Humans 40(6): 1310-1328, DOI: 10.1109/TSMCA.2010.2049993.

Takagi, T. and Sugeno, M. (1985). Fuzzy identification of systems and its application to modeling and control, IEEE Transaction on System, Man and Cybernetics SMC15(1): 116-132.

Van Huffel, S. and Lemmerling, P. (Eds.) (2002). Total Least Squares and Errors-in-Variables Modeling: Analysis, Algorithms and Applications, 1st Edn., Springer-Verlag, London.

Xu, D., Jiang, B. and Shi, P. (2012). Nonlinear actuator fault estimation observer: An inverse system approach via a T-S fuzzy model, International Journal of Applied Mathematics and Computer Science 22(1): 183-196, DOI: 10.2478/v10006-012-0014-9.

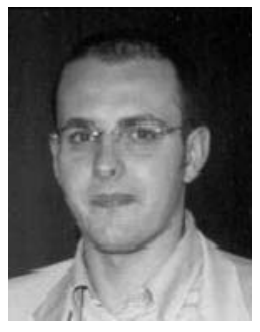

Silvio Simani was born in Ferrara in 1971 He received the "Laurea" degree (cum laude) in electrical engineering in 1996 from the Department of Engineering at the University of Ferrara (Italy), and in 2000 the Ph.D. in information science (automatic control) at the University of Ferrara and Modena (Italy). Since 2006 he has been an IEEE senior member, and since 2000 a member of the SAFEPROCESS Technical Committee. Since 2002 he has been an assistant professor at the Department of Engineering, University of Ferrara. His research interests include fault diagnosis and fault tolerant control, and system identification. He is the author of about 100 refereed journal and conference papers, as well as two books on these topics.

Received: 19 April 2012 Revised: 30 September 2012 Re-revised: 9 January 2013 